To appear in Vehicle System Dynamics

Vol. 00, No. 00, Month 20XX, 1-24

\title{
Gyroscopic stabilisers for powered two-wheeled vehicles
}

\author{
R. Lot and J. Fleming \\ Mechanical Engineering Department, University of Southampton (UK) \\ (received $X X X X 20 X X)$
}

\begin{abstract}
This paper illustrates the potential of a gyroscopic stabiliser for the stabilisation of single track vehicles, at low and high speed as well as during braking. Alternative systems are considered, including single and twin counter-rotating gyroscopes, spinning and precessing with respect to different axes, either freely (passive stabilisers) or in a controlled way (active stabilisers). A suitable mathematical model has been developed and stability has been investigated both by eigenvalue calculation and time domain simulations. It has been found that the most effective configuration is one where the gyroscope(s) spin with respect to an axis parallel to the wheels' spin axis and swing with respect to the vehicle yaw axis. Passive systems may effectively stabilise both weave and wobble at medium and high speed, but cannot stabilise the vehicle at low and zero speed. On the contrary, actively controlled gyroscopes are capable of stabilising the vehicle in its whole range of operating speed, as well as during braking. The alteration of the original vehicle handling characteristics is negligible when active counterrotating gyroscopes are used, and still acceptable if a single gyroscope is adopted instead.
\end{abstract}

Keywords: motorcycles; powered two wheelers; single-track vehicles; stability; gyroscopic stabilisation.

\section{Introduction}

It is well known that motorcycles and single track vehicles in general may suffer from serious stability problems [1-4]. More specifically, there are three typical modes that may become unstable depending on both the vehicle characteristics and the motion conditions: capsize, weave, and wobble. The Capsize mode is stable at null and low speed and becomes unstable after the minimum speed threshold has been passed. The Weave mode consists of oscillation of the whole vehicle at a frequency between 2 and $4 \mathrm{~Hz}$, it is unstable at low speed, becoming stable as the speed increases but finally it may become unstable again at high speed. Finally, the Wobble mode mainly consists of the oscillation of the steering assembly, typically with a frequency between 5 and $9 \mathrm{~Hz}$, and may become unstable in the medium speed range. Such stability problems are even more pronounced under acceleration and braking [2,3], or while cornering [3].

Instability is certainly one of the reasons that collision avoidance systems, automated emergency braking, and other similar active safety technologies have not yet been developed for motorcycles, while they are already available for cars. There are at least two technologies that may be exploited for stabilisation and active safety of motorcycles: steer-by-wire and gyroscopic stabilisation. In a steer-by-wire system, the direct mechanical connection between the handlebars and the front wheel is replaced by an electro-

\footnotetext{
* Corresponding author. Email: roberto.lot@soton.ac.uk
} 
mechanical system which actively controls the wheel steer angle. While some details may be found in [5], a discussion about this topic is out of scope of the present article, which focuses on gyroscopic stabilisation only. Early references for gyroscopic stabilisers for automotive applications date back to the $19^{\text {th }}$ century [6], followed by similar patents at the beginning of the $20^{\text {th }}$ century. The stabilisation of the roll of motorcycle by the precession of a gyro rotor of high moment of inertia spinning at high speed is claimed by [7], similar ideas are proposed by [8] for a toy motorcycle application. In recent times, [9-11] claim the idea of using a couple of identical gyroscopes spinning and precessing in opposite directions, so a gyroscopic torque effect is generated only when the precession motion is activated Gyroscopes may also be used for regenerative braking [9, 10]. Despite this practical interest in the subject, the scientific literature on this topic is scarce and incomplete. In [12], a gyroscopic stabiliser consisting of two identical gyroscopes, counter-rotating and mechanically connected to swing in opposite directions has been used to stabilise a bicycle. A simple mathematical model of the vehicle and a simple control algorithm have been used to develop a prototype, which was successfully tested at very low speed (up to $1 \mathrm{~m} / \mathrm{s}$ ). In [13], a gyroscopic stabiliser consisting of a single gyroscope spinning with respect to a vertical axis and swinging with respect to a pitch axis to stabilise the roll motion of a single track vehicle called Ecomobile has been studied. A simplified model of the dynamics of a two-wheeled vehicle that considers only the lateral position of the contact point and the roll angle was employed to develop a simple control system capable of stabilising the roll motion. The effects of steering angle, yaw motion, and tyre-road interactions are neglected and the control moment produced by the gyroscope is assumed to be directly controllable. An analog stabiliser system has been studied [14], where a more complex model that considers curvature of the vehicle's path is given in, but again the effects of steering and tyre dynamics are ignored.

The aim of this paper is to provide a general understanding of the gyroscopic stabilisation of single track vehicles by making a systematic analysis of the different aspects of the problem. Different stabiliser configurations, consisting of either one or two counter-rotating gyroscopes and swinging with respect to different axis are analysed and discussed. Both actively controlled stabilisers and passively precessing gyroscopes are considered. The influence that gyroscopic stabilisers have on capsize, weave and wobble is specifically addressed, as is the stabiliser's effect on vehicle handling.

The paper is organised as follows: first, different alternatives to generate either a roll, a yaw, or a mixed roll-yaw gyroscope torque are illustrated first (section 2). Then, a mathematical model describing the dynamics of the combined vehicle-gyroscope system is then introduced (section 3). Finally, the performance of both passive stabilisers (section 4 ) and active ones (section 5) are assessed. Finally, results are summarised and discussed in section 6 .

\section{Gyroscopic torque generation}

Different configurations of gyroscopic stabilisers have been considered in the existing literature, including single gyroscopes $[7,8,13,14]$ and twin counter-rotating gyroscopes [9-12], which spin either with respect to a nominal yaw $[7,9,10,13,14]$, pitch $[7,8]$ and even roll [11] axis. However, the advantages and disadvantages of these different configurations are unclear, and there is no comparative analysis of their stabilisation performance. For this reason, this section introduces the principles of gyroscopic torque generation and compares all sensible configurations of one and two gyroscopes that may be used to generate a stabilising effect on a motorcycle. 


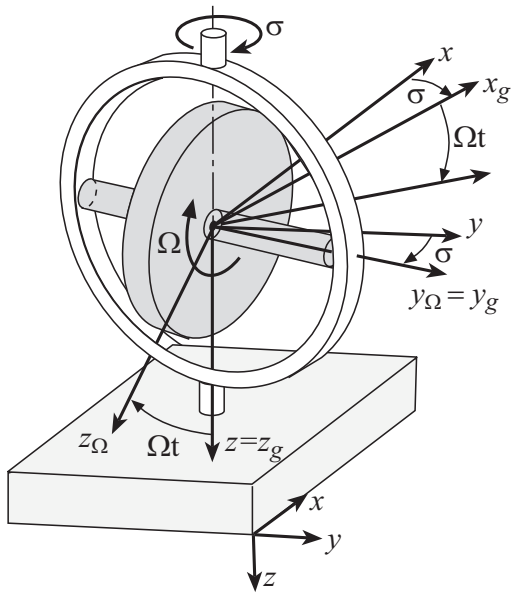

(a) The reference frame $(x, y, z)$ is attached to the base, the frame $\left(x_{g}, y_{g}, z_{g}\right)$ is attached to the swinging gimbal, and the frame $\left(x_{\Omega}, y_{\Omega}, z_{\Omega}\right)$ is attached to the spinning gyroscope.

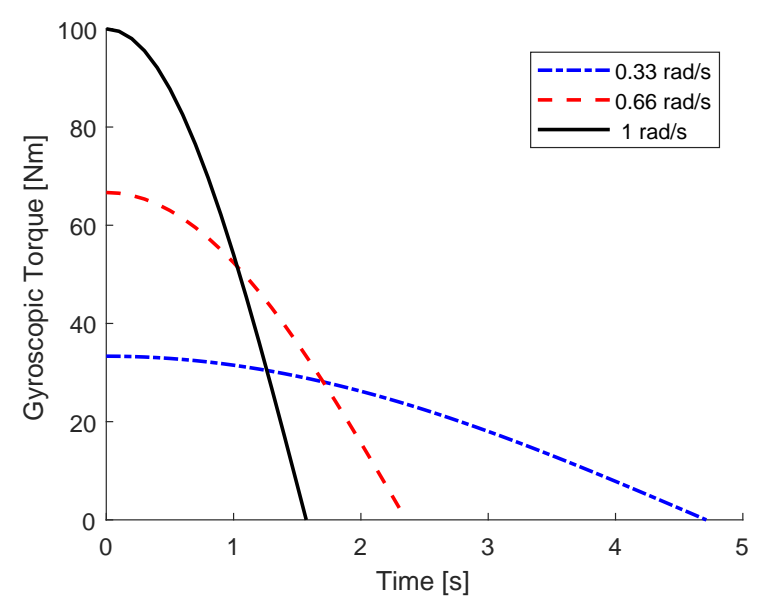

(b) Gyroscopic torque $G$ for different swinging velocities (angular momentum $I_{g} \Omega=100 \mathrm{Nms}$ ).

Figure 1. Gyroscopic torque generated by a gyroscope mounted on a gimbal.

The simplest way to generate a gyroscopic torque is depicted in Figure 1a and consists of a gimbal which swings by an angle $\sigma$ with respect to the vertical axis $z$, while supporting a gyroscope with spins with constant velocity $\Omega$ with respect to the $y_{g}$ axis. The gyroscope is an axially-symmetric body and its inertia tensor is a diagonal matrix $\boldsymbol{I}=\operatorname{diag}\left(I_{d}, I_{g}, I_{d}\right)$, where $I_{y y}=I_{g}$ is the axial (spinning) inertia and $I_{x x}=I_{z z}=I_{d}$ are the diametral inertias. By defining $\boldsymbol{\omega}$ as the angular velocity of the gimbal and $\boldsymbol{\Omega}=(0,-\Omega, 0)^{T}$ as the gyroscope spinning velocity relative to the gimbal, the angular momentum of the gyroscope is:

$$
\boldsymbol{K}=\boldsymbol{I}(\boldsymbol{\omega}+\boldsymbol{\Omega})
$$

Because of the axial symmetry of the gyroscope, this expression is valid both in the reference frame $\left(x_{\Omega}, y_{\Omega}, z_{\Omega}\right)$ attached to the gyroscope and in the reference frame $\left(x_{g}, y_{g}, z_{g}\right)$ attached to the gimbal. The latter is more convenient for the derivation of Euler's equations, which read:

$$
\frac{\mathrm{d} \boldsymbol{K}}{\mathrm{d} t}=\boldsymbol{I} \dot{\boldsymbol{\omega}}+\boldsymbol{\omega} \times \boldsymbol{I}(\boldsymbol{\omega}+\boldsymbol{\Omega})=\boldsymbol{M}
$$

where $M$ is the moment vector of active and reactive external forces with respect to the gyroscope centre of mass and $\dot{\boldsymbol{\Omega}}=0$ by assumption. The gyroscope torque is by definition:

$$
G=\omega \times I \Omega
$$

By assuming that the spinning velocity is much greater than the angular velocity of the gimbal $\Omega \gg|\boldsymbol{\omega}|$, the term $\boldsymbol{\omega} \times \boldsymbol{I} \boldsymbol{\omega}$ (which is quadratic in the angular speeds $\omega_{i} \omega_{j}$ ), Euler's equations may be simplified as follows:

$$
I \dot{\omega}+G=M
$$


For a fixed base, the angular velocity of the gimbal is simply $\boldsymbol{\omega}=(0,0, \dot{\sigma})^{T}$ and Euler's equations become:

$$
\begin{gathered}
I_{g} \Omega \dot{\sigma}=M_{x}^{g} \\
0=M_{y}^{g} \\
I_{d} \ddot{\sigma}=M_{z}^{g}
\end{gathered}
$$

where the suffix $g$ highlights that vectorial equation (4) has been projected onto the reference frame $\left(x_{g}, y_{g}, z_{g}\right)$ attached to the gimbal. In equation (5), $G=I_{g} \Omega \dot{\sigma}$ is the gyroscopic torque, which is orthogonal to both the spinning and swinging axes; $M_{y}^{g}$ is the (null) torque necessary to maintain the gyroscope spin motion and $M_{y}^{g}$ is the torque necessary to control the swing motion. The projection of the gyroscopic torque into the reference frame $(x, y, z)$, which is attached to the base, reads:

$$
\boldsymbol{G}=\left(\begin{array}{c}
G_{x} \\
G_{y} \\
G_{z}
\end{array}\right)=\left(\begin{array}{c}
I_{g} \Omega \dot{\sigma} \cos \sigma \\
I_{g} \Omega \dot{\sigma} \sin \sigma \\
0
\end{array}\right)
$$

For the purpose of roll stabilisation, only the component $G_{x}$ of the gyroscopic torque is useful. This torque increases with the angular speed $\dot{\sigma}$ but decreases with the angle $\sigma$. Therefore, even if a high torque can be generated by a fast swing motion, a continuous torque may be generated only for a short time (see Figure 1b). Indeed, the integral of the torque $G_{x}$ for a complete swing rotation, i.e. from maximum torque ( $\left.\sigma=0\right)$ to null torque $(\sigma=\pi / 2)$, is always equal to the angular momentum of the gyroscope:

$$
\int_{0}^{T} G_{x} d t=I_{g} \Omega \int_{0}^{T} \dot{\sigma} \cos \sigma d t=\left.I_{g} \Omega \sin \sigma\right|_{0} ^{\pi / 2}=I_{g} \Omega
$$

Now we consider the application of a swinging gyroscope on a motorcycle which moves with angular speed $\boldsymbol{\omega}_{m}=\left(\omega_{x}, \omega_{y}, \omega_{z}\right)^{T}$. Consequently, the gimbal angular speed is $\boldsymbol{\omega}=$ $\left(\omega_{x}, \omega_{y}, \omega_{z}+\dot{\sigma}\right)^{T}$ in the reference frame attached to the vehicle chassis (see Figure 2a, $\alpha=0$ ), while in the reference frame attached to the gimbal itself is:

$$
\boldsymbol{\omega}=\left(\begin{array}{c}
\omega_{x} \cos \sigma+\omega_{y} \sin \sigma \\
-\omega_{x} \sin \sigma+\omega_{y} \cos \sigma \\
\omega_{z}+\dot{\sigma}
\end{array}\right)
$$

By introducing expression (17) into equation (3), one obtains the following expression of the roll gyroscopic torque:

$$
\boldsymbol{G}_{\phi}=\left(\begin{array}{c}
G_{x} \\
G_{y} \\
G_{z}
\end{array}\right)=\left(\begin{array}{c}
\left(\omega_{z}+\dot{\sigma}\right) \cos \sigma \\
\left(\omega_{z}+\dot{\sigma}\right) \sin \sigma \\
-\left(\omega_{x} \cos \sigma+\omega_{y} \sin \sigma\right)
\end{array}\right) I_{g} \Omega
$$

where components $G_{x}, G_{y}, G_{z}$ are defined in the chassis reference frame. Equation 9 shows that the gyroscopic roll torque $G_{x}$ includes not only the term generated by the swing motion $\dot{\sigma}$, but also a term in $\omega_{z}$ that cannot be controlled directly. Additionally, the angular velocity $\omega_{x}$ generates a gyroscopic moment on the $z$ axis, so that the gyroscope creates a cross coupling between angular motion of the motorcycle about the $x$ and $z$ 


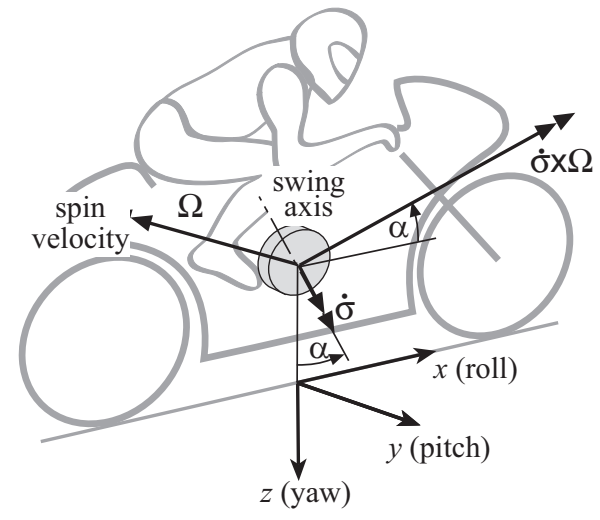

(a) Gyroscope spinning with respect to the $y$ axis, gimbal swinging with respect to an axis in the $x y$ plane.

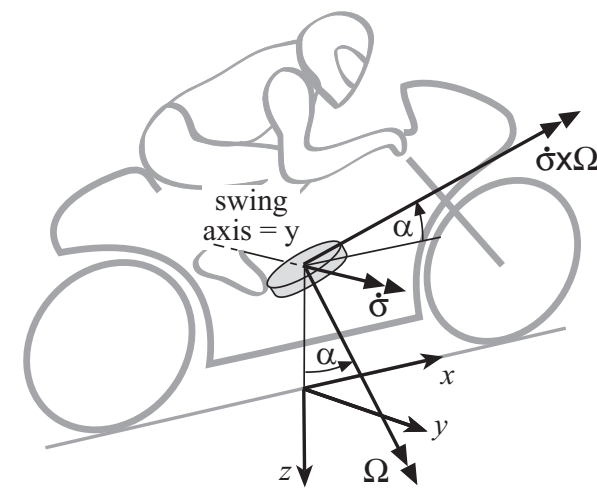

(b) Gyroscope spinning with respect to an axis in the $x y$ plane, gimbal swinging with respect to the $y$ axis.

Figure 2. Equivalent layouts that generate the same gyroscopic torque.

axes. There are also some coupling terms between the $y$ and $z$ axes. These terms are proportional to $\sin \sigma$ and therefore less significant than the previous ones.

An alternative way to generate a roll gyroscopic torque is to utilise a gyroscope which spins with respect to the yaw axis $z$ and swings with respect to the pitch axis $y$ (Figure $2 \mathrm{~b}$, assuming $\alpha=0$ ). In this case the gyroscopic torque reads:

$$
\boldsymbol{G}_{\phi}^{\prime}=\left(\begin{array}{c}
\left(\omega_{y}+\dot{\sigma}\right) \cos \sigma \\
\omega_{z} \sin \sigma-\omega_{x} \cos \sigma \\
-\left(\omega_{y}+\dot{\sigma}\right) \sin \sigma
\end{array}\right) I_{g} \Omega
$$

The gyroscope now creates a pitch-roll coupling between $x$ and $y$ axis. This situation is quite different from the previous one: while the yaw-roll gyroscopic cross-terms (9) act in addition to the already coupled motorcycle dynamics, the roll-pitch gyroscopic crossterms (10) create an ex-novo coupling between in-plane and out-of-plane motion, which is much less predictable and potentially more dangerous. For example, the pitch motion generated during braking, or while passing over a bump, will create a roll gyroscopic torque which may capsize the vehicle. For this reason, we believe that this configuration is unsuitable for stabilising purposes and will not be considered further in this paper.

However, for both the pitch-spinning and yaw-spinning configurations, it is possible to almost cancel the cross-coupling effects by using a pair of counter-rotating gyroscopes. More precisely, by using two equal gyroscopes $I_{g 1}=I_{g 2}=I_{g} / 2$ with opposite spin and swing rotations $\Omega_{1,2}= \pm \Omega, \sigma_{1,2}= \pm \sigma$, the expression of the gyroscopic torques (9) and (10) both reduce to:

$$
\boldsymbol{G}_{\phi}^{\prime \prime}=\left(\begin{array}{c}
\dot{\sigma} \cos \sigma \\
\omega_{z} \sin \sigma \\
-\omega_{y} \sin \sigma
\end{array}\right) I_{g} \Omega
$$

The cross-coupling between $x$ and $z$ axes has been completely eliminated and the roll torque depends on the swing motion only. $G_{z}$ still depends on the pitching angular rate, but for small swing angles this term is small too.

Motorcycle stability is not only related to the roll motion, for example weave stability 
is heavily associated to the yaw motion. Therefore, a yaw gyroscopic torque may in principle be used to improve stability. A yaw gyroscopic torque may be generated by using a gyroscope which spins with respect to the pitch axis $y$ and swings with respect to the roll axis $x$ (Figure 2a, assuming $\alpha=\pi / 2$ ). The complete expression of the gyroscopic torque is:

$$
\boldsymbol{G}_{\psi}=\left(\begin{array}{c}
\omega_{z} \cos \sigma-\omega_{y} \sin \sigma \\
\left(\omega_{x}+\dot{\sigma}\right) \sin \sigma \\
-\left(\omega_{x}+\dot{\sigma}\right) \cos \sigma
\end{array}\right) I_{g} \Omega
$$

Alternatively a gyroscope which spins with respect to the roll axis $x$ and swings with respect to the pitch axis $y$ may be used (Figure 2a, assuming $\alpha=\pi / 2$ ), the corresponding gyroscopic effect is:

$$
\boldsymbol{G}_{\psi}^{\prime}=\left(\begin{array}{c}
-\left(\omega_{x}+\dot{\sigma}\right) \sin \sigma \\
\left(\omega_{x} \sin \sigma+\omega_{z} \cos \sigma\right) \\
-\left(\omega_{x}+\dot{\sigma}\right) \cos \sigma
\end{array}\right) I_{g} \Omega
$$

The gyroscopic torques (12) and (13) contain not only the swing gyroscopic term $I_{g} \Omega \dot{\sigma}$, which is generated purposefully and may be controlled, but also some additional crosscoupling terms which cannot be controlled directly. For the pitch-spinning gyroscope (12), the main cross-coupling is between the yaw and roll motion (with a minor coupling between pitch and yaw), while for the the roll-spinning gyroscope (13), the main crosscoupling is between the yaw and pitch motion (plus a minor coupling between roll and yaw). As discussed for the roll torque case, the gyroscopic coupling between between in-plane and out-of-plane motion has to be avoided because it is highly unpredictable and potentially dangerous, so the second configuration will not be considered further.

Once again, the utilisation of two equal counter-rotating and counter-swinging gyroscopes makes it possible to cancel the main cross-coupling terms, and the gyroscopic torque becomes:

$$
G_{\psi}^{\prime \prime}=\left(\begin{array}{c}
-\omega_{y} \sin \sigma \\
\omega_{x} \sin \sigma \\
-\dot{\sigma} \cos \sigma
\end{array}\right) I_{g} \Omega
$$

expression which is valid for both the $x$ - and $y$-axis spinning layouts.

It is useful to generalise the results discussed above to consider the generation of a gyroscopic torque which has both roll and yaw components. For a couple of pitchspinning gyroscopes swinging by an axis inclined by the angle $\alpha$ with respect to the yaw axis (Figure 2a), the gyroscopic torque on the chassis may be calculated as follows:

$$
\begin{gathered}
\boldsymbol{G}=\boldsymbol{G}_{\phi} \cos \alpha+\boldsymbol{G}_{\psi} \sin \alpha \\
\boldsymbol{G}=\left(\begin{array}{c}
\dot{\sigma} \cos \sigma \cos \alpha-\omega_{y} \sin \sigma \sin \alpha \\
\left(\omega_{x} \sin \alpha+\omega_{z} \cos \alpha\right) \sin \sigma \\
-\left(\omega_{y} \sin \sigma \cos \alpha+\dot{\sigma} \cos \sigma \sin \alpha\right)
\end{array}\right) I_{g} \Omega+\frac{i_{1}+i_{2}}{2}\left(\begin{array}{c}
\omega_{z} \cos \sigma \\
\dot{\sigma} \sin \sigma \\
-\omega_{x} \cos \sigma
\end{array}\right) I_{g} \Omega
\end{gathered}
$$

where $i_{1}, i_{2}$ indicates the spinning and swinging directions of the gyroscopes. In other words $i_{1,2}=1$ represents the single gyroscope configuration, while $i_{1,2}= \pm 1$ represents the twin counter-rotating gyroscope configuration. In the latter case, the equation 
remains valid also in the case of gimbals swinging with respect to the pitch axis and gyroscopes spinning with respect to an axis inclined by the angle $\alpha$ with respect to the yaw axis, see Figure 2b.

We now discuss how to control the gyroscope precession motion. The simplest way to constraint the gyroscope motion is to use a torsional spring $k_{s}$ and a damper $c_{s}$ on the swing axis (passive control). Alternatively, an actuator providing torque $T_{\sigma}$ may be used to actively control the gyroscope precession. Both possibilities are included in the following equation of swinging motion:

$$
\begin{aligned}
& I_{d} \ddot{\sigma}+c_{s} \dot{\sigma}+k_{s} \sigma+\frac{i_{1}+i_{2}}{2} I_{d}\left(\dot{\omega}_{x} \sin \alpha+\dot{\omega}_{z} \cos \alpha\right)= \\
& T_{\sigma}+\left(\omega_{x} \cos \alpha-\omega_{z} \sin \alpha\right) \cos \alpha I_{g} \Omega+\frac{i_{1}+i_{2}}{2} \omega_{y} \sin \alpha I_{g} \Omega+M_{\omega}
\end{aligned}
$$

where $M_{\omega}$ is a (minor) quadratic angular velocity term, which reads:

$$
\begin{aligned}
M_{\omega} & =\left(I_{g}-I_{d}\right)\left[\left(\omega_{x} \cos \alpha-\omega_{z} \sin \alpha\right)^{2}-\omega_{y}^{2}\right] \sin \alpha \cos \alpha \\
& +\frac{i_{1}+i_{2}}{2}\left(I_{g}-I_{d}\right) \omega_{y}\left(\omega_{x} \cos \alpha-\omega_{z} \sin \alpha\right)\left(\sin ^{2} \alpha-\cos ^{2} \alpha\right)
\end{aligned}
$$

The main figure of merit for design of the gyroscopic stabiliser is the angular momentum $I_{g} \Omega$, as the gyroscopic torque generated on the chassis is proportional to this value. Higher values of spinning angular velocity $\Omega$ are therefore desirable as they allow a given angular momentum to be realised with a lower moment of inertia $I_{g}$, and therefore reduced size and weight for the gyroscope. The present study uses $I_{g} \Omega=100 \mathrm{Nms}$, which corresponds to either a single gyroscope of mass $10 \mathrm{~kg}$ and radius $11 \mathrm{~cm}$, or twin gyroscopes of mass $5 \mathrm{~kg}$ and radius $8 \mathrm{~cm}$, spinning at $30000 \mathrm{rpm}$. We estimate that the gimbal assembly will double the required mass. The gyroscope may be installed, for example, beneath the saddle, but it will function correctly regardless of its placement on the rear chassis.

\section{Dynamics of the single track vehicle with gyroscopes}

This section illustrates the mathematical model that will be used for analysing the influence of the gyroscope system on the vehicle stability.

Since both the rider and the stabiliser concurrently control the motorcycle, their interaction will also be considered.

Riders control the motorcycle by using different control inputs: throttle and brakes are used to control the vehicle speed and acceleration (longitudinal motion control), while steer torque and leaning of the upper body are used to control the stability and direction of the vehicle (lateral motion control). Expert and race riders are also capable of coordinating the use of longitudinal and lateral controls, for example they are capable of coordinating steer and throttle control inputs while exiting a curve. In this paper, the assessment of gyroscopic stabiliser performance is limited to the fundamental case of lateral motion control. In 1979, Aoki [15] carried out an experimental study in order to assess the relative significance of steer torque and rider lean control. Based on the analysis of the yaw velocity transfer function, Aoki concluded that riders control the motorcycle primarily in terms of steer torque, while body leaning control is negligible. In 1988, another experimental study by Katayama et al. [16] found again that that steer torque is the primary control, while the upper body is kept vertical but does not really contribute to the control. Other studies have confirmed this tendency, and a comprehen- 
sive critical discussion of the literature may be found in [17]. Accordingly, several authors have modelled rider control as a steer torque only [18-23] while some have considered the lean control too $[20,24,25]$. Recently, thanks to the availability of commercial multibody software, more complex rider models have been proposed. For example, a virtual rider made up of 15 rigid bodies which has 28 degrees of freedom is described in [26]. However, in practice it is difficult to identify rider parameters to populate such models and there are no experimental studies which confirm their superiority to simpler steer and lean rider models.

With regards to stability, in 1971 Sharp [1] introduced a now classical four degrees of freedom (roll, yaw, steer, and lateral velocity) mathematical model which is capable of reproducing the characteristic modes of motorcycles, namely weave, wobble, and capsize. Relaxation equations are used for tyre forces. The predictions of this model are in fair agreement with experimental results [27-29], however further research has demonstrated that frame compliance [30-32] and in particular fork bending stiffness [29] impact stability too. Suspension does not affect straight running stability, but in cornering outof-plane modes (weave, wobble, capsize) and in-plane modes (heave, pitch, and wheels hop) are coupled and hence suspension characteristics may influence lateral stability too [3]. Finally, the stability of a motorcycle is also influenced by the rider's passive behavior. In [32], a dynamical model where the riders upper torso has a rotational degree of freedom is proposed. In [33] the riders arms are also modelled, while in [16] the riders upper and lower body are added to Sharps motorcycle model [1]. As shown in [29, 34, 35], the correlation between the predictions of such models and experimental tests is reasonably good. A more complex model, which takes into account not only the leaning motion of the riders upper torso but also the connection of the rider arms with the handlebars has been presented in [25].

It is evident that the rider is simultaneously an active subject, which is capable of controlling vehicle direction and rollover stability, and a passive one whose characteristics influence weave and wobble stability. Indeed, from a biomedical perspective, the neuromuscular behavior of human riders is characterised by three main components: (i) the cognitive response of the brain, (ii) the stretch reflex response, and (iii) the pure passive (intrinsic) response [36]. The voluntary riding task is associated to the rider cognitive response, which in turn comprises two parts: a feed-forward action for which the frequency content can reach significant values and a feed-back action with a bandwidth of approximately $1-2 \mathrm{~Hz}[36,37]$. On the contrary, both the (ii) stretch reflex and (iii) passive intrinsic responses are involuntary and passive, and as a first approximation they may be modelled with an elastic spring and a viscous damper respectively [36], in accordance with experimental observation $[16,34,35]$.

In accordance with the previous discussion, a mathematical model has been defined that aims to appropriately reproduce the control capability of the rider and evaluate the stability of the motorcycle, while avoiding any unessential over-complication. The motorcycle model is similar to the one proposed by Sharp [1], but includes five rigid bodies: front chassis, rear chassis, rider, and wheels. However, unlike in Sharp [1] the five degrees of freedom (roll $\phi$, yaw $\psi$, steer $\delta$, longitudinal and lateral velocity $u, v$ ) are not linearised and the full non-linear dynamics are considered. The tyre model has been improved by including the cross-sectional curvature radius $\rho[38]$ and tyre contact forces and torques are modelled according to the non-linear magic formula [38, 39], as shown in Figure 4. The motorcycle suspecnsion has not been considered, as they would add considerable complexity to the model while their influence on the out-of-plane dynamics is secondary.

The gyroscopic torque generated by the stabiliser is applied to the motorcycle chassis 


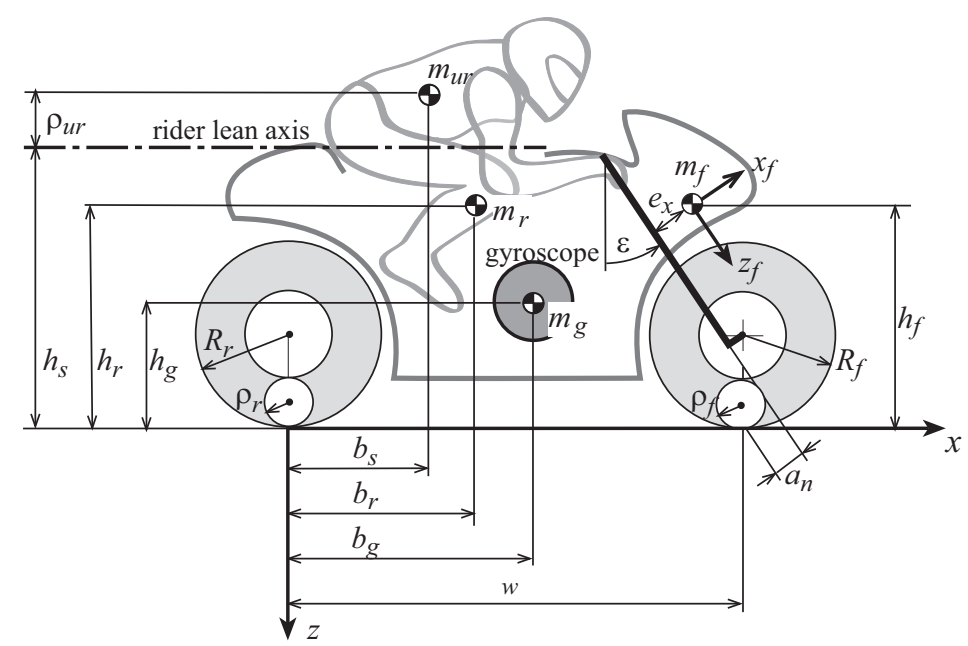

Figure 3. Motorcycle geometry and mass distribution

by considering equation (15), where angular velocity $\boldsymbol{\omega}$ is calculated in terms of yaw, roll, and pitch angles as follows:

$$
\boldsymbol{\omega}=\left(\begin{array}{c}
\omega_{x} \\
\omega_{y} \\
\omega_{z}
\end{array}\right)=\left(\begin{array}{c}
\cos (\mu) \dot{\phi}-\sin (\mu) \cos (\phi) \dot{\psi} \\
\sin (\phi) \dot{\psi}+\dot{\mu} \\
\sin (\mu) \dot{\phi}+\cos (\mu) \cos (\phi) \dot{\psi}
\end{array}\right)
$$

Similarly, expression (17) is introduced into the swinging equation (16). Rider control inputs include both steer torque $T_{\delta}$ and lean body torque $T_{\theta}$, in other words the human body has been modelled as two rigid bodies: the lower rider, which is rigidly attached to the chassis, and the upper rider, which rotates by the angle $\theta$ with respect to an horizontal leaning axis, Figure 3 . A rotational spring $k_{\theta}$ and a damper $c_{\theta}$ have also been connected to the lean axis in order to simulate the passive leaning motion, with parameter values corresponding to the natural frequency and damping ratio given in Table 3 in [36]. The Maple-based toolbox MBSymba [40, 41] was used to derive the non-linear equations of motion, which are quite complex and therefore have not been reported in full. However, while the non-linear model is used for time domain simulations, the model has been linearised in straight running conditions before carrying out stability analysis and calculation of frequency response functions, in conclusion the linearised equations of motion of the whole system may be expressed as a descriptor state space system:

$$
\mathbf{E} \dot{\boldsymbol{x}}=\mathbf{A}(t) \boldsymbol{x}+\mathbf{B} \boldsymbol{u}
$$

The state $\boldsymbol{x}$ is a 12-dimensional vector:

$$
\boldsymbol{x}=\left(v, \omega_{\psi}, \omega_{\phi}, \omega_{\delta}, Y_{r}, Y_{f}, \phi, \delta, \omega_{\sigma}, \sigma, \omega_{\theta}, \theta\right)^{T}
$$

consisting of the lateral speed $v$, yaw rate $\omega_{\psi}$, roll and steer velocities $\omega_{\phi}, \omega_{\delta}$, front and rear tyre lateral forces $Y_{f}, Y_{r}$, roll and steer angles $\phi, \delta$ swing velocity and rotation $\omega_{\sigma}, \sigma$ rider lean velocity and rotation $\omega_{\theta}, \theta$.

The input vector is made up of three variables

$$
\boldsymbol{u}=\left(T_{\delta}, T_{\sigma}, T_{\theta}\right)^{T}
$$


namely the rider steering torque $T_{\delta}$, the gyroscope(s) control torque $T_{\sigma}$, and the rider body leaning torque $T_{\theta}$.

The square matrix $\mathbf{E}$ is constant in time and symmetric:

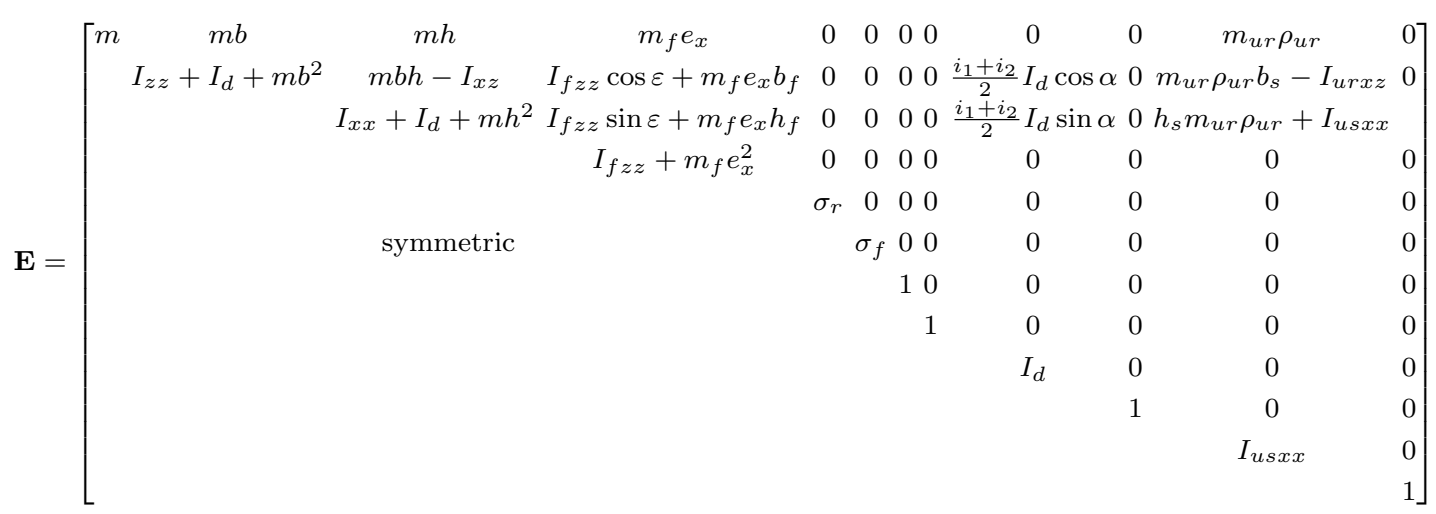

Square matrix $\mathbf{A}(t)$ is time-varying in general due to dependencies on longitudinal velocity $u$ and acceleration $\dot{u}=a_{x} . \mathbf{A}(t)$ is non symmetric and sparse, non-zero terms are:

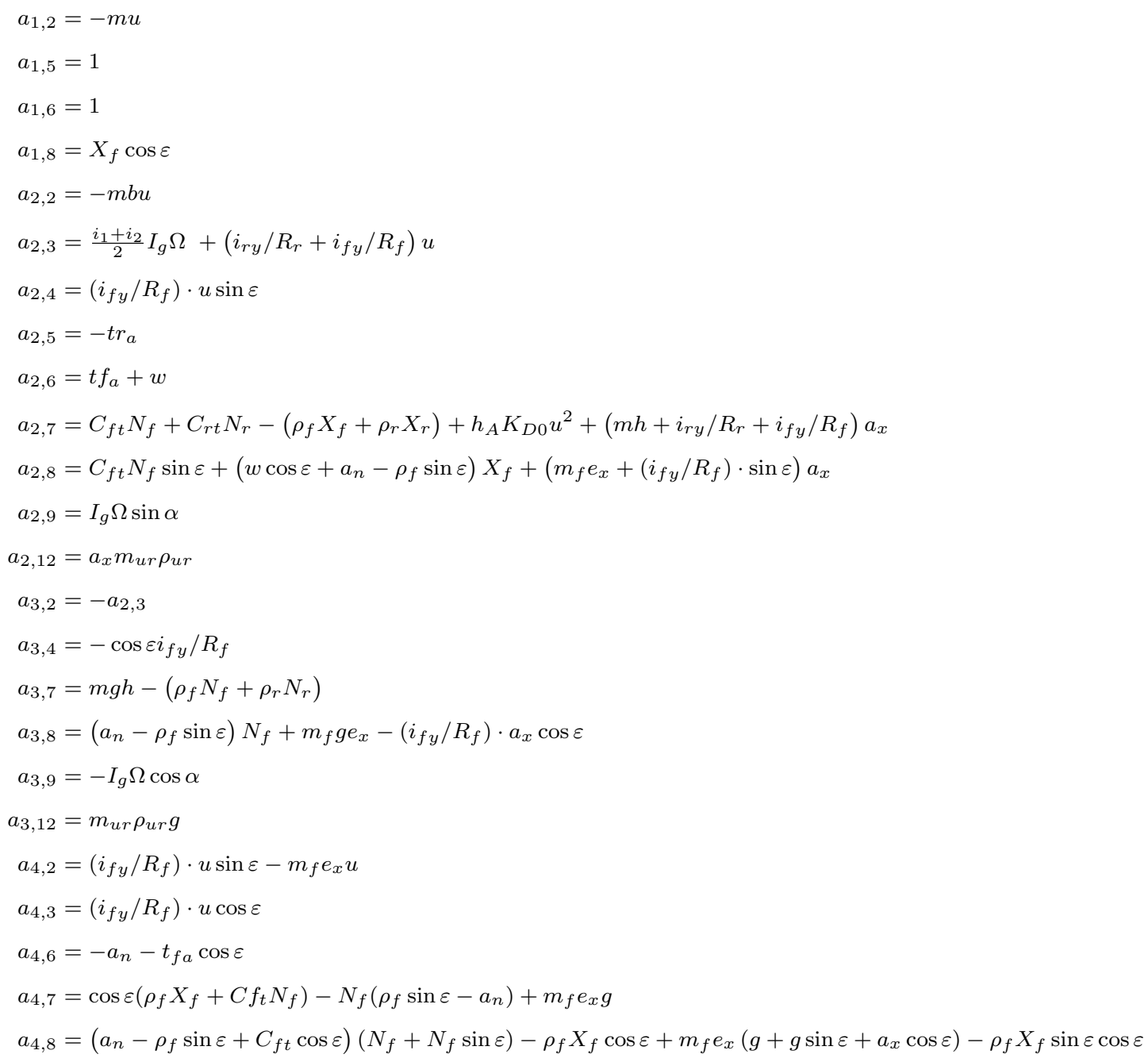




$$
\begin{aligned}
a_{5,1} & =-N_{r} C r_{\lambda} \\
a_{5,5} & =-u \\
a_{5,7} & =C r_{\phi} N_{r} u \\
a_{6,1} & =-N_{f} C f_{\lambda} \\
a_{6,2} & =-w N_{f} C f_{\lambda} \\
a_{6,4} & =-a_{n} N_{f} C f_{\lambda} \\
a_{6,6} & =-u \\
a_{6,7} & =N_{f} C f_{\phi} u \\
a_{6,8} & =\left(C_{f \phi} \sin \varepsilon+C_{f \lambda} \cos \varepsilon\right) N_{f} u \\
a_{7,3} & =1 \\
a_{8,4} & =1 \\
a_{9,2} & =-a_{2,9} \\
a_{9,3} & =-a_{3,9} \\
a_{9,9} & =-C_{d} \\
a_{9,9} & =-K_{d} \\
a_{10,9} & =1 \\
a_{11,2} & =-m_{u r} \rho_{u r} u \\
a_{11,7} & =m_{u r} \rho_{u r} g \\
a_{11,11} & =-C_{u r} \\
a_{11,11} & =-m_{u r} \rho_{u r} g-K_{u r} \\
a_{12,11} & =1
\end{aligned}
$$

The input matrix $\mathbf{B}$ has size $12 \times 3$, the only non-zero elements are

$$
\begin{aligned}
b_{4,1} & =1 \\
b_{11,2} & =1 \\
b_{9,3} & =1
\end{aligned}
$$

in order to provide steer torque, gyroscope swing torque, and rider lean torque inputs respectively. Vehicle parameters are given in table Table 1, moreover non-linear tyre characteristics (which are used in time simulations only) are reported in Figure 4.

\section{Passive gyroscopic stabilisation}

In this section we discuss how to improve vehicle stability by means of gyroscopes either rigidly mounted to or elastically suspended on the chassis, without employing any active system to control the precession motion of the gyroscopes.

The loci of the complex eigenvalues of the motorcycle as the velocity varies between 0.5 (indicated by the circle marker) and $80 \mathrm{~m} / \mathrm{s}$ are highlighted in Figure 5a. The capsize mode is stable throughout all of the speed range, although the damping is negligible at medium and high speeds. The wobble mode has a frequency range of $7-10 \mathrm{~Hz}$ and is unstable above $53 \mathrm{~m} / \mathrm{s}$, while the weave mode has a frequency range of $0-3 \mathrm{~Hz}$ and in unstable below $7.2 \mathrm{~m} / \mathrm{s}$. In particular, weave is coupled to the upper rider body lean 
Table 1. Motorcycle, tyre, gyroscope and rider parameters

\begin{tabular}{|c|c|c|c|}
\hline parameters & value & & description \\
\hline$w$ & 1.413 & $m$ & wheelbase \\
\hline$e$ & 0.0408 & $m$ & eccentricity \\
\hline$\varepsilon$ & 21.2 & $\circ$ & castor angle \\
\hline$a_{n}$ & 0.112 & $m$ & mechanical trail \\
\hline$c_{\delta}$ & 6 & $N m / r a d s^{-1}$ & steering column damping \\
\hline$C_{D} A$ & 0.35 & $m^{2}$ & drag coefficient \\
\hline$m_{f}, m_{r}$ & $39,216.2$ & $k g$ & front and rear chassis mass \\
\hline$\left(b_{f}, h_{f}\right)$ & $(1.38,0.506)$ & $m$ & $x-y$ position of front centre of mass \\
\hline$\left(b_{r}, h_{r}\right)$ & $(0.522,0.538)$ & & $x-y$ position of rear centre of mass \\
\hline$I_{f x x}, I_{f y y}, I_{f z z}$ & $4.57,4.72,0.781$ & $\mathrm{kgm}^{2}$ & front principle moments of inertia \\
\hline$I_{r x x}, I_{r y y}, I_{r z z}$ & $12.4,32.1,27.2$ & $\mathrm{kgm}^{2}$ & rear principle moments of inertia \\
\hline$R_{f}, R_{r}$ & $0.319,0.3$ & $m$ & front and rear tyre radius \\
\hline$\rho_{f}, \rho_{r}$ & $0.06,0.105$ & $m$ & tyre cross-sectional radius of curvature \\
\hline$\sigma_{f}, \sigma_{r}$ & $0.165,0.21$ & $m$ & front and rear relaxation length \\
\hline$C_{f \lambda}, C_{f \phi}$ & $9.68,0.91$ & - & front tyre stiffnesses \\
\hline$C_{r \lambda}, C_{r \phi}$ & $13.7,1.20$ & - & rear tyre stiffnesses \\
\hline$C_{f a}, C_{r a}$ & $0.407,0.383$ & - & self-aligning moment coefficients \\
\hline$C_{f t}, C_{r t}$ & $0.023,0.023$ & - & overturning moment coefficients \\
\hline$I_{g}$ & 0.0318 & $\mathrm{kgm}^{2}$ & axial moment of inertia \\
\hline$I_{d}$ & 0.0175 & $\mathrm{kgm}^{2}$ & diametral moment of inertia \\
\hline$m_{g}$ & 10 & $\mathrm{~kg}$ & gyroscope mass \\
\hline$\left(b_{g}, h_{g}\right)$ & $(0.6,0.7)$ & & $x-y$ position of centre of mass \\
\hline$\Omega$ & 30000 & $R P M$ & spinning angular velocity \\
\hline$m_{u r}$ & 35 & $k g$ & rider upper body mass \\
\hline$I_{\text {urxx }}$ & 2.8 & $\mathrm{kgm}^{2}$ & upper body moment of inertia \\
\hline$\left(b_{s}, h_{s}\right)$ & $(0.7,0.75)$ & $m$ & $x-y$ position of centre of mass \\
\hline$\rho_{u r}$ & 0.2 & $m$ & radius of pivot from centre of mass \\
\hline
\end{tabular}
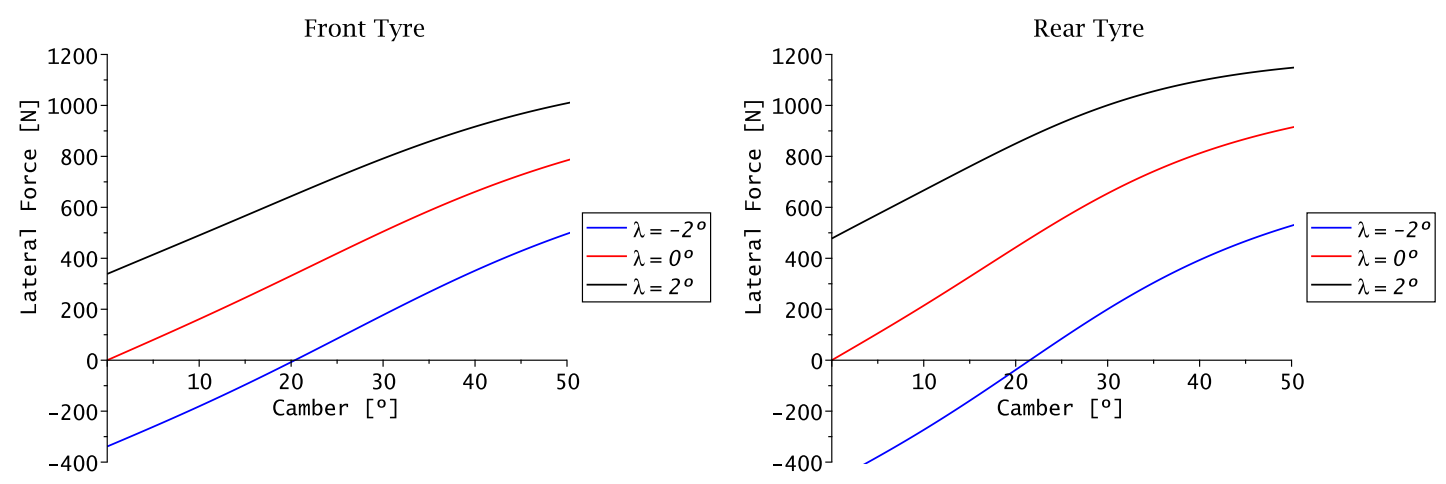

Figure 4. Lateral tyre force vs camber angle, for different value of sideslip $\alpha$ and vertical load of $1000 \mathrm{~N}$

motion, so the well-known weave pattern discussed in [1] is here split into two separate modes. The rider stretch reflex and intrinsic response have only a modest stabilising effect on these modes. Figure 5 also displays the root loci when a gyroscope spinning in the same direction as the rear wheel and having angular momentum $I_{g} \Omega=100 \mathrm{Nms}$ is rigidly attached to the chassis. This is essentially equivalent to increasing the inertia of the rear wheel, with the only difference being that the gyroscopic effect is not correlated to the vehicle speed, and, as noted in [1], this improves the stability of weave mode at high speeds. Indeed, this has stabilised the oscillatory behaviour of the weave mode occurring 


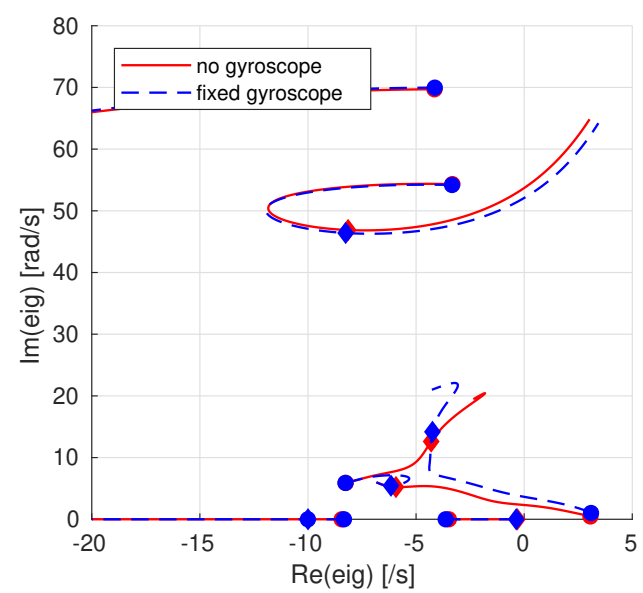

(a) Fixed gyroscope(s)

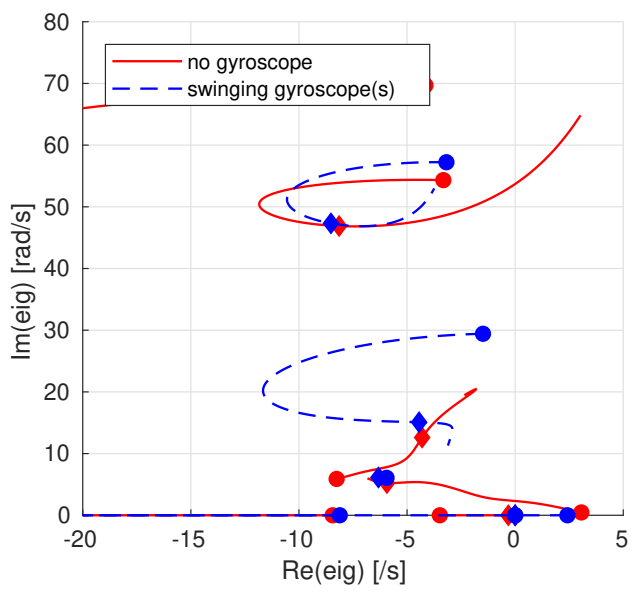

(b) Free swinging gyroscope(s)

Figure 5. Root loci, speed varying from 0.5 to $80 \mathrm{~m} / \mathrm{s}$ (circle marker at $0.5 \mathrm{~m} / \mathrm{s}$, diamond marker at $20 \mathrm{~m} / \mathrm{s}$ ).

at an angular frequency of approximately $\omega=20 \mathrm{rad} / \mathrm{s}(f=3.2 \mathrm{~Hz})$. If, while spinning with respect to the $y$ pitch axis, the gyroscope is allowed to swing freely with respect to the $z$ yaw axis (free precession), the behaviour is radically different as shown in Figure 5b. The unstable oscillatory behaviour of both weave and wobble modes at high speeds has been stabilised by the freely-swinging gyroscope. The behaviour of the weave mode is also very different from before: the frequency of oscillation now decreases with increasing speed, and another non-oscillatory mode has appeared that is unstable at low speeds, in place of the low-speed instability of the weave. Interestingly, when the single gyroscope is replaced by a twin gyroscope system, i.e. a system composed by two identical gyroscopes with spins in opposite directions that are also mechanically connected in order to swing in opposite directions, the eigenvalues remain the same. Finally, a parametric analysis varying the gyroscope angular momentum from 0 to $200 \mathrm{Nms}$ has been carried out, finding similar behavior which is of course more pronounced as the gyroscope angular momentum increases.

Although the stabilisation of the oscillatory modes at high speeds is promising, the freely-swinging gyroscope is unpractical because the gyroscopes have no equilibrium position. Therefore, we have considered adding a spring and a damper which partially restrains the swinging motion of the gyroscope(s). In particular, Figure 6a gives eigenvalue loci for a range of values of spring constant, with no damping considered. The presence of the spring seems to have only a minor effect on the wobble mode of the motorcycle, and this effect is apparent at high speeds. However there is a large change in the behaviour of the weave mode. As the spring constant is increased, the frequency of oscillation of the weave mode also increases, and the damping at medium speeds is increased. However, for values of spring constant above approximately $400 \mathrm{Nm} / \mathrm{rad}$, the stabilising effect of the free gyroscope at high speed is lost. In other words, stabilisation requires a "soft" spring rather than a "hard" one (Figure 5a). To ensure wobble stability, we have therefore chosen a spring constant of $k_{s}=160 \mathrm{Nm} / \mathrm{rad}$, which corresponds to a natural frequency of approximately $32 \mathrm{~Hz}$ for the gyroscope swinging motion. The effect of adding a damper to the swing dynamics of the gyroscope is demonstrated in Figure $6 \mathrm{~b}$, where the damping value $c_{s}=3.35 \mathrm{Nms} / \mathrm{rad}$ corresponds to critical damping in the left hand side of equation (16). It may be observed that this damping further stabilises the weave mode at low to medium speeds. Figure $6 \mathrm{c}$ shows the root loci for different 


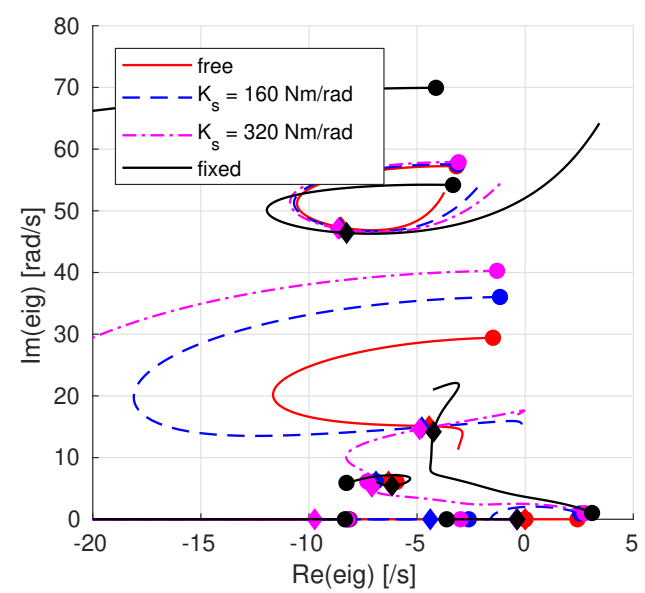

(a) Effect of spring stiffness, undamped system.

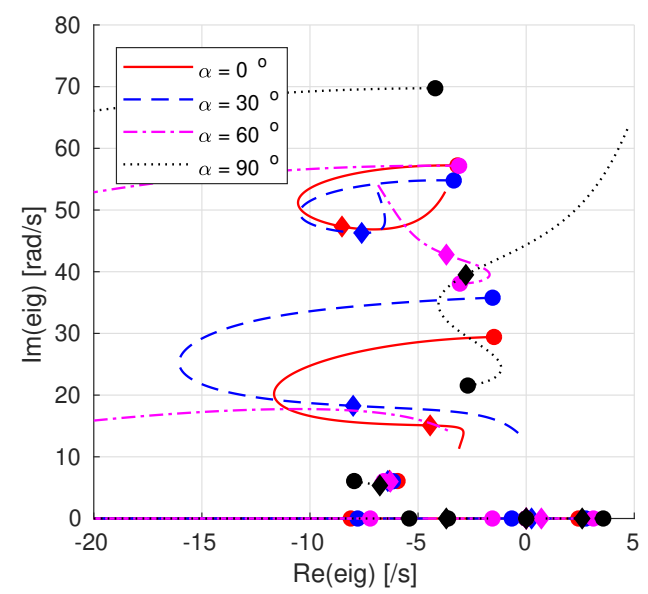

(c) Swing axis orientation $\left(k_{s}=0\right)$

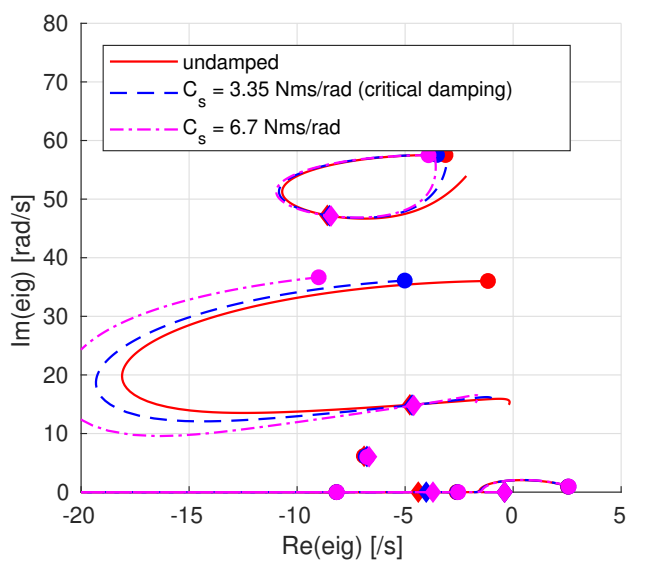

(b) Effect of damping, stiffness $k_{s}=160 \mathrm{Nm} / \mathrm{rad}$

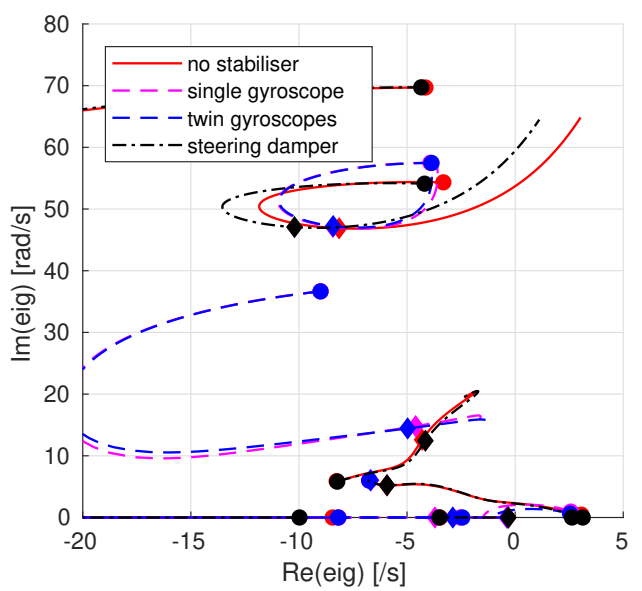

(d) Comparison of stabilising strategies $\left(k_{s}=160\right)$

Figure 6. Root loci (speed varying from 0.5 to $80 \mathrm{~m} / \mathrm{s}$ ).

orientations of the gyroscope swing axis, from $\alpha=0^{\circ}$ (vertical swing axis) to $\alpha=90^{\circ}$ (horizontal swing axis). Since we have previously found that only soft springs may ensure the gyroscopic stabilising effect, we have considered only the unrestrained case $k_{s}=0$ for simplicity. The high-speed stabilisation of the oscillatory modes is retained in both the $30^{\circ}$ and $60^{\circ}$ loci but lost in the $90^{\circ}$ locus, so we may conclude that $\alpha$ in the range $0-60^{\circ}$ will stabilise the motorcycle. Beyond this point, the weave mode is no longer stable. Additionally, increasing the angle of the swinging axis is seen to increase the frequency of the motorcycle's weave mode at low to medium speeds. Recalling that $\alpha=0^{\circ}$ corresponds to the generation of gyroscopic roll torque, and $\alpha=90^{\circ}$ the generation of yaw torque, we may conclude that the wobble mode may be stabilised by either roll or yaw gyroscopic torques, but the weave mode only by gyroscopic roll torque. Finally, Figure 6d compares the stability of the final gyroscopic stabiliser design with the original motorcycle. In the same figure, we also compare the effect of the stabiliser with the more straightforward approach of increasing the damping on the steering column. the value of the damper for this comparison was chosen as $c_{\delta}=9$, which gives similar damping of the wobble mode around $60 \mathrm{~m} / \mathrm{s}$ to the gyroscopic stabiliser. We see that the gyroscope may be used to stabilise both the wobble and weave modes of the bike. The more common method of 

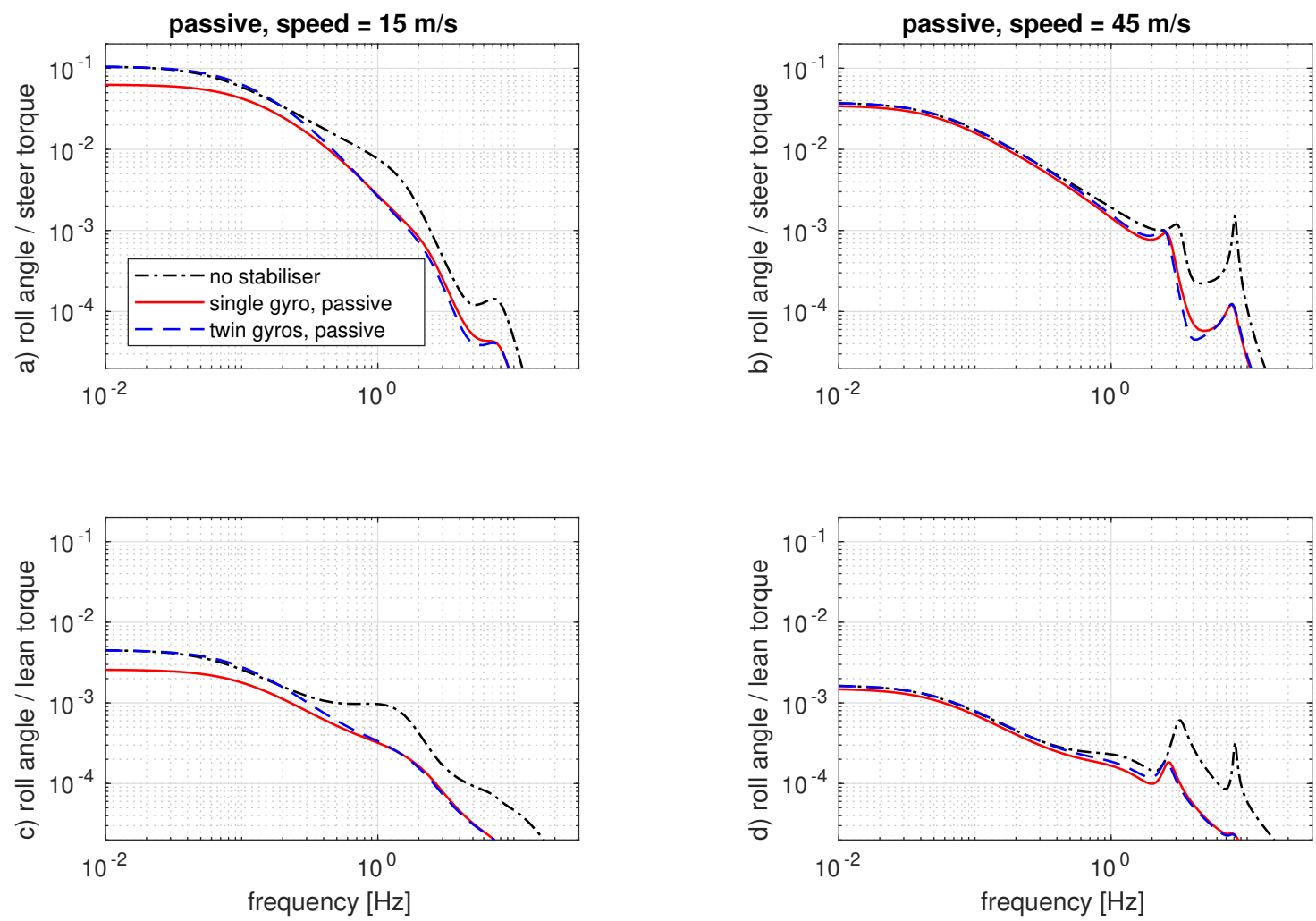

Figure 7 . Frequency response functions with passive stabilisation.

applying damping to the steering column is beneficial for stabilising the wobble mode but is slightly detrimental to weave-mode stability. The figure also shows that, in term of vehicle stabilisation, single and twin counter-rotating gyroscopes are almost equivalent.

The dynamic response of the motorcycle is now analysed in terms of roll angle frequency response functions, which have been identified as good metrics for the assessment of motorcycle handling [42]. Figure $7 \mathrm{a}$ and $7 \mathrm{~b}$ depict the motorcycle roll response to the rider steering torque input at medium $(15 \mathrm{~m} / \mathrm{s})$ and high speeds $(45 \mathrm{~m} / \mathrm{s})$ respectively for three different configurations: i) without any stabiliser, ii) with a single gyroscopic stabiliser, and iii) with twin counter-rotating gyroscopes. At low frequencies, the single gyroscope configuration ii) manifests a small gain reduction in comparison to the original configuration i), which implies that an increased steady-state steering torque is required when cornering. However, this problem may be eliminated by adopting the twin gyroscope configuration iii). However, for frequencies of $1 \mathrm{~Hz}$ and higher both stabilizers ii) and iii) exhibit similar behavior in which there is some extra attenuation at medium to high frequencies, corresponding to the slightly slower system response during transient maneuvers such as lane change or entering a curve. With regard to the vehicle speed, high speed gains are generally lower than low speed ones. Weave and wobble resonances are evident at high speed but have higher damping when a gyroscopic stabiliser is present, which is consistent with the root loci depicted in Figure 6d)

Figure $7 \mathrm{c}$ and $7 \mathrm{~d}$ depict the motorcycle roll response to the rider lean torque input, for the same configurations as just discussed for the steer torque input. It may be observed that, although the differences between stabilised and original motorcycle exhibit the same trends seen for the steer input, lean torque gains are smaller than steer torque gains by a factor of at least 10 (or more) for frequencies up to $1 \mathrm{~Hz}$, with a greater difference 
visible at lower frequencies. As a result of this low magnitude the practical possibility of controlling the vehicle via lean torque is minimal. It is also worth recalling that only the feed-forward rider action may include high frequency content, while feed-back actions have a bandwidth limited to a maximum of $1-2 \mathrm{~Hz}[36,37]$. It may be concluded that that steer torque control is largely more effective than lean motion control, in accordance with former experimental studies $[15,16]$

So far we have demonstrated that a passive gyroscope can have a beneficial effect on the weave and wobble stability of a motorcycle; moreover the influence of a gyroscopic stabiliser on motorcycle handling has been analysed in term of frequency response functions. We now analyse the non-linear behavior of the motorcycle, both in transient and steady-state condition, by means of time domain simulations. In particular, we must verify that the stabilised motorcycle has acceptable cornering performance. This is a particular concern as the gyroscope produces a torque on the chassis that opposes roll motion, hence also opposing the natural tendency of the rider to bank into a corner. The reference maneuver consists of leaning the motorcycle from straight running into cornering with a final roll angle of $40^{\circ}$ (i.e. a lateral acceleration of $a_{y}=6.3 \mathrm{~m} / \mathrm{s}^{2}$ ) in approximately $1 \mathrm{~s}$, then after a further $2 \mathrm{~s}$ the motorcycle is straightened up again. Since the focus is on motorcycle handling rather than rider ability, the steering action is modelled as a simple proportional-derivative controller that applies a torque on the handlebars, while the rider upper body lean motion is inhibited because it is less important. The original and stabilised motorcycles responses are compared in Figure 8. While using a single gyroscopic stabiliser, the roll angle response appears only a little slower than when a stabiliser is not present, although the required steering torque increases. However, the differences are modest and the usual counter-steering behaviour is retained in all configurations. The peak value of the swing angle during the manoeuver is $17^{\circ}$, which is considerable but not excessive considering that the maneuver is quite challenging with a maximum roll angle of $40^{\circ}$ and a peak roll rate of $46^{\circ} / \mathrm{s}$. However, the magnitude of the precession motion may be reduced by introducing a non-linear spring, so that identical behaviour is obtained when the system is linearised, but a larger torque is applied for large deviations of the gyroscope from its nominal position. For example, by using a non-linear spring $T_{s}=k_{\sigma}\left(1+\sigma^{2} / \sigma_{0}^{2}\right) \sigma$ with $\sigma_{0}=0.02 \mathrm{~m}$ it has been found that the peak swing angle is decreased to $3.3^{\circ}$. The cornering response is almost unaffected by this change, so it has not been added to the figure.

In conclusion, we have demonstrated that a passive gyroscope system may be used to stabilise the weave and wobble modes of the motorcycle. The single or twin gyroscope system should swing with respect to the $z$ yaw axis, vehicle stability is sensitive mainly to the angular moment of the gyroscope and swinging spring stiffness. However, a passively precessing gyroscopic system is not capable of fully stabilising the low speed capsize. This is not surprising and may be seen from a straightforward argument based on the principle of conservation of energy - a rollover motion of the motorcycle lowers its centre of mass and hence its gravitational potential energy. It is therefore impossible to return an initially stationary, but rolled, motorcycle to an upright position without an external energy input. For a moving motorcycle this energy input can be provided by the longitudinal motion of the vehicle, but below some critical speed this becomes impossible and an active control system must be employed, as discussed in the next section. 

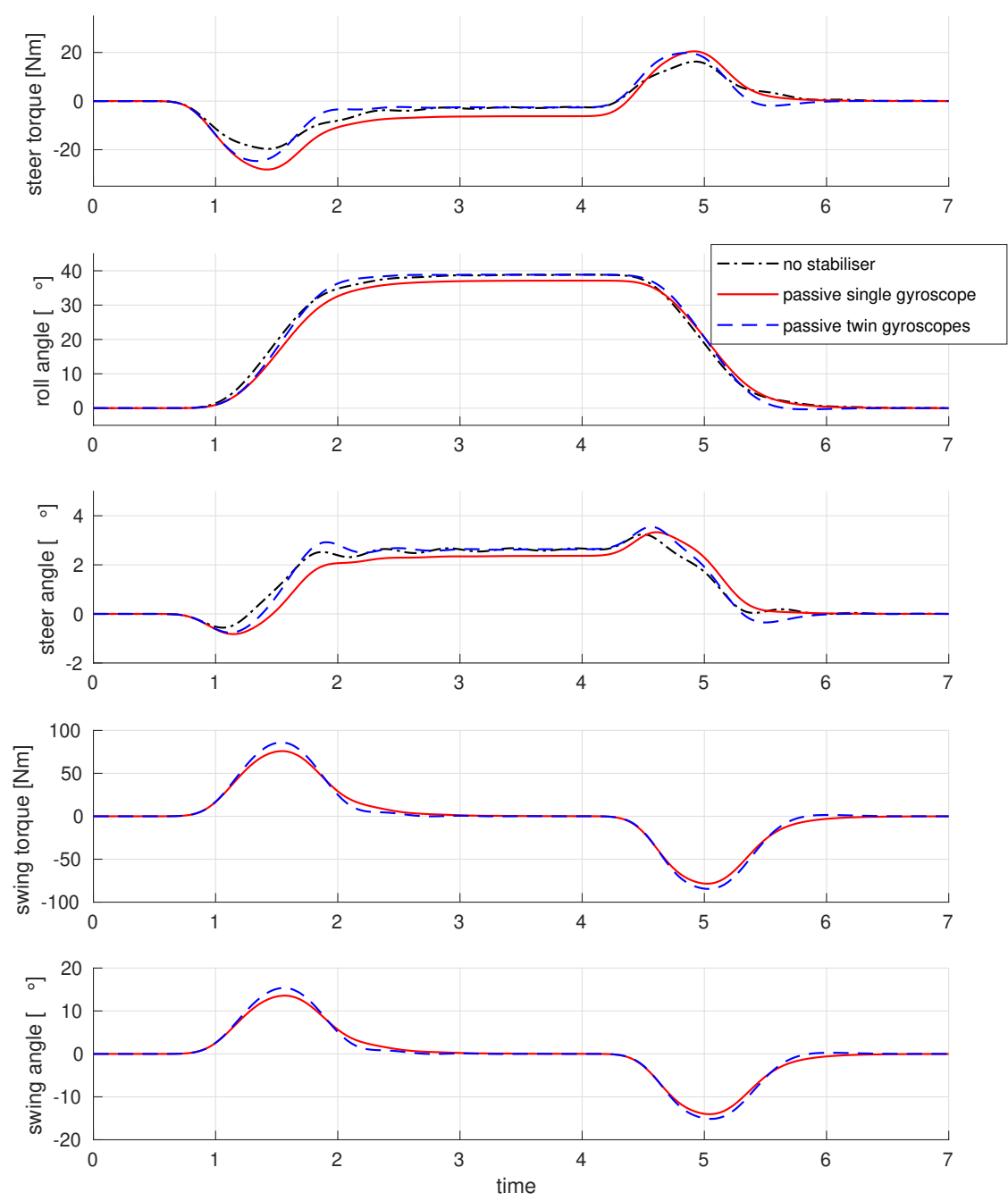

Figure 8. Motorcycle response under cornering, with passive gyroscopic stabiliser (motorcycle speed $u=15 \mathrm{~m} / \mathrm{s}$, steady cornering acceleration $a_{y}=6.3 \mathrm{~m} / \mathrm{s}^{2}$, transient roll rate peak $\omega_{\phi, \text { max }}=46^{\circ} / \mathrm{s}$ ).

\section{Active gyroscopic stabilisation}

In this section we discuss the possibility of using an active system that controls the swing motion of the gyroscope(s) to further improve vehicle stability. This is particularly important for stabilisation at low speed that, as discussed at the end of the previous section, cannot be guaranteed by a passive system.

For control design, it is unreasonable to assume that the value of rider lean and lean rate is available to the active gyroscope system. Therefore for control design we consider the simplified system for which the lean angle and lean rate are identically zero and the state reduces to:

$$
\boldsymbol{x}=\left(v, \omega_{\psi}, \omega_{\phi}, \omega_{\delta}, Y_{r}, Y_{f}, \phi, \delta, \omega_{\sigma}, \sigma\right)^{T}
$$


Table 2. Setpoints and penalty coefficients for gain-scheduled controllers

\begin{tabular}{c|cc}
$u_{i}$ & $q_{i}$ & $r_{i}$ \\
\hline 80 & $10^{6}$ & 1 \\
16 & $10^{6}$ & 1 \\
4 & 1 & $10^{-3}$ \\
0.5 & 1 & $10^{-3}$
\end{tabular}

Hence, the reduced matrices $\mathbf{A}_{r}$ and $\mathbf{B}_{\mathbf{r}}$ used for controller design correspond to the top-left $10 \times 10$ block of $\mathbf{A}$ and the top 10 rows of $\mathbf{B}$ respectively. We stress that this reduced system is used for controller design only and the full model is used to analyse system performance.

As the principal objective is to understand the potential of gyroscopic stabilisers, we consider the design of a full-state feedback controller, where the torque applied to the gyroscope is a linear function of the current state vector:

$$
T_{\sigma}=-\boldsymbol{K} \boldsymbol{x}
$$

The matrix $\boldsymbol{K}$ is chosen to correspond to the infinite-horizon linear quadratic regulator (LQR) for the cost function

$$
J=\int_{0}^{\infty}\left(\phi^{2}+q \sigma^{2}+r T_{\sigma}^{2}\right) d t
$$

where $q$ is a tuning parameter that penalises the swing angle, and $r$ denotes a tuning parameter that penalises swing input torque. This has the advantage that one may compare different system configurations (such as using a single or twin gyroscope, or different orientations) in a straightforward and unified way by retaining the same cost function for each case.

Since system eigenvalues vary with the speed and longitudinal acceleration, a constant set of gains $\boldsymbol{K}$ is not capable of stabilising the vehicle in the whole range of operating conditions. To solve this problem, feedback gains are linearly interpolated between gainscheduled controllers defined by a feedback matrix $K_{i}$ and a speed setpoint $u_{i}$, which are given in Table 2 together with the corresponding cost function weights. At the low speed setpoints $(0.5 \mathrm{~m} / \mathrm{s}$ and $4 \mathrm{~m} / \mathrm{s})$ an equal penalty has been given to both the swing and roll angle, while a large input penalty is chosen in order to stabilise the system using as little swing torque as possible. At higher speeds (the $16 \mathrm{~m} / \mathrm{s}$ and $80 \mathrm{~m} / \mathrm{s}$ setpoints) any swing motion is heavily penalised instead. In this way the gyroscope motion is constrained by the controller and the motorcycle has similar high-speed dynamics to when the gyroscope is not present.

The result of applying this controller to the system is shown in Figure 9 in terms of the root loci of the controlled system. Figure 9a shows the eigenvalues of the motorcycle with active gyroscopic stabilisation (but without rider control, $T_{\delta}=0$ ) for a speed varying from $0.5 \mathrm{~m} / \mathrm{s}$ and $80 \mathrm{~m} / \mathrm{s}$. The wobble mode stabilisation is analogue to the one obtained with the passive system, figure $6 \mathrm{~d}$. Moreover, low speed capsize and weave have now been stabilised, see Figure 9b. Because the vehicle rollover stabilisation is particularly important during braking, this situation has been analysed too. In [43] and [44], it is shown that the time-varying linear system (18) is stable when the associated frozen-time eigenvalues are negative, and the rate of change of $\boldsymbol{A}(t)$ is sufficiently slow. Therefore, eigenvalue analysis is used here to assess braking stability as is also done, for example, in [2-4]. Without changing the control gains, the beneficial effect of this active stabilisation is clearly visible in the whole speed range, as visible in Figure 10a-b. 


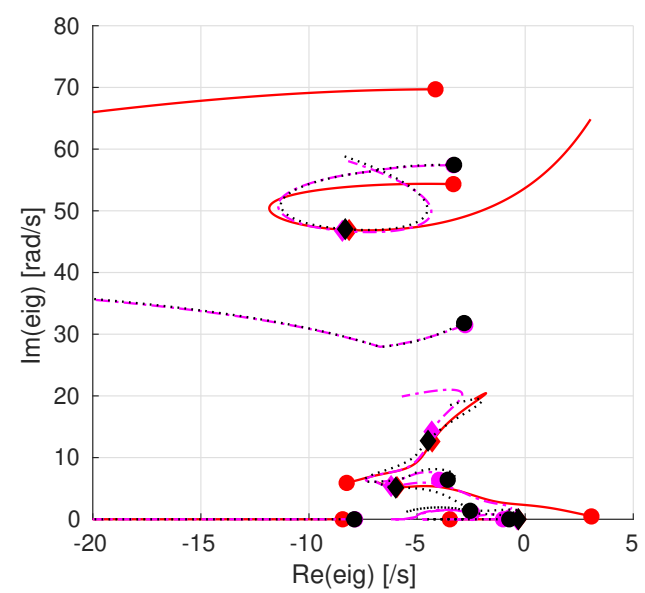

(a) Root loci (overview)

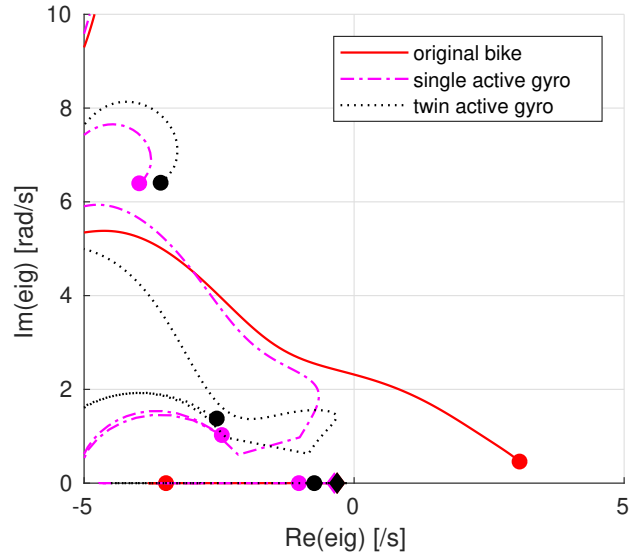

(b) Root loci (zoom)

Figure 9. Root loci, constant speed motion (different speed between 0.5 and $80 \mathrm{~m} / \mathrm{s}$ ).

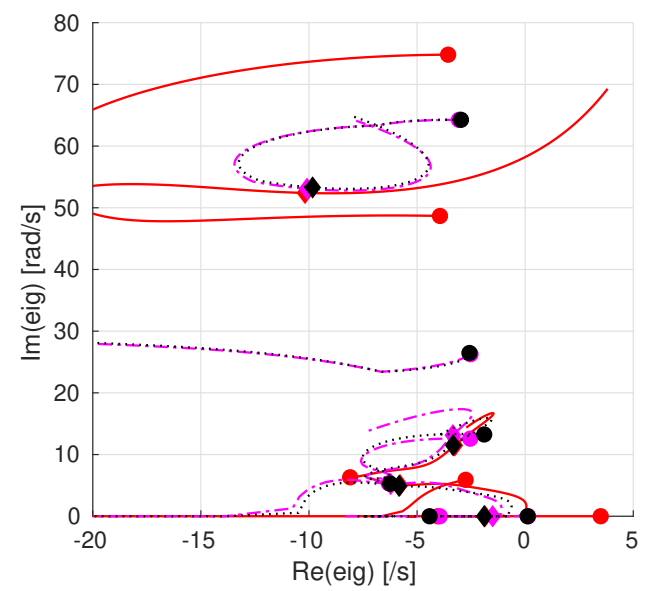

(a) Root loci (overview)

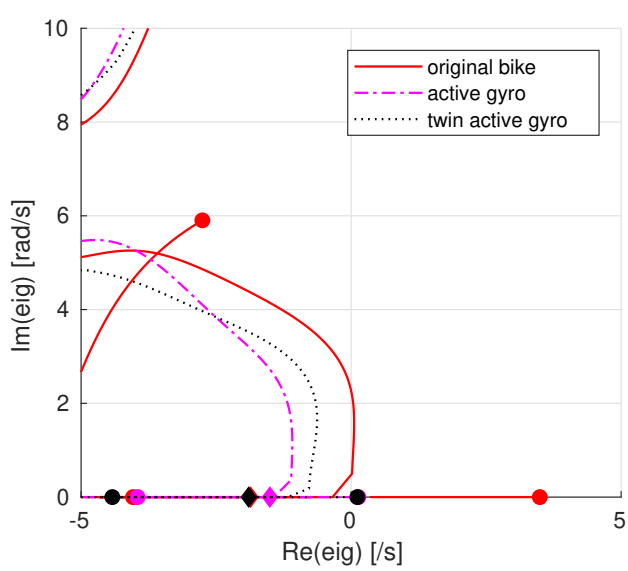

(b) Root loci (zoom)

Figure 10. Root loci, braking with constant deceleration $4 \mathrm{~m} / \mathrm{s}^{2}$ (speed between 0.5 and $80 \mathrm{~m} / \mathrm{s}$ ).

The dynamics behavior of the motorcycle may be further discussed by inspecting frequency response functions (FRFs). The vehicle now has three separate, independent inputs: the steer and leaning torque, which are both controlled by the rider, plus the gyroscope swing torque. Figure 11a shows the magnitude of the steer torque to roll angle response, calculated when the gyroscope control loop is closed. As in the passive case (figure 7a), when using a single gyroscope it is visible that the DC gain of this transfer function is reduced, again corresponding to the increased steer torque required in the cornering simulation when using only a single gyroscope. At a frequency of $1 \mathrm{~Hz}$ and above, the magnitude response between the original vehicle and the stabilised one is practically the same, therefore the influence of the stabiliser in fast transient manoeuvres will be negligible. The figure also shows that the adoption of twin counter-rotating gyroscopes makes it possible to approximately maintain the original vehicle handling characteristics. Figure 11b shows the upper-body lean torque to roll angle response, calculated when the gyroscope control loop is closed. While the presence of the stabilisers does not significantly affect the frequency response functions, lean to roll gains remain 

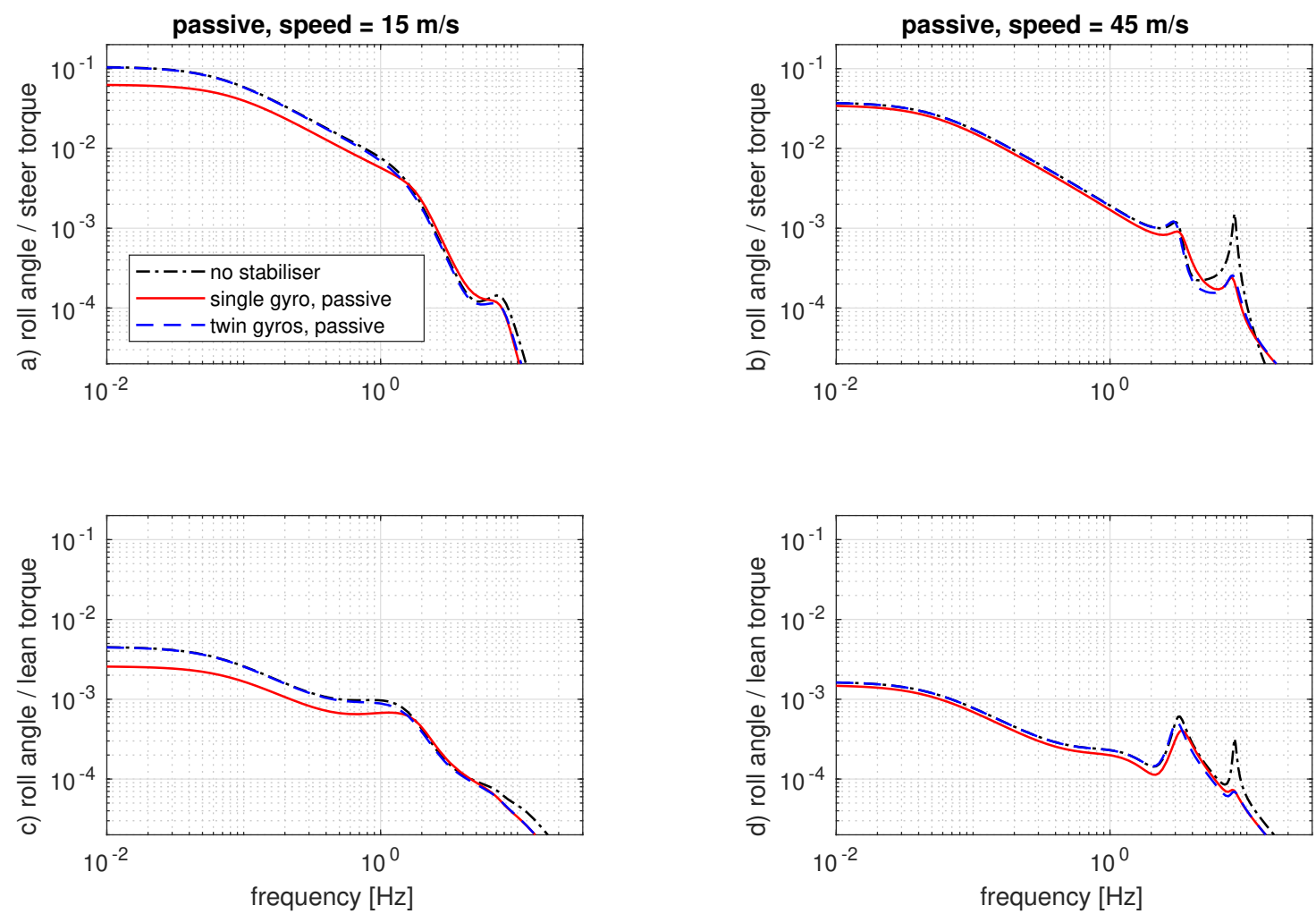

Figure 11. Frequency response functions with active stabilisation.

sensibly smaller than steer to roll gains. Therefore, as already seen for passive stabilisers, body lean control is almost irrelevant compared to steer control. Figure 11c shows the magnitude of the swing torque to roll angle response, calculated without any rider control action (hands-off) steering torque is. This transfer function approximates an integrator at the low to medium frequencies, shown. Moreover it includes a small resonance peak at high frequencies (although this is well above the range of interest for handling). At very low-frequency, the magnitude of the swing-to-roll FRF is greater than the magnitude of the steer-to-roll FRF, suggesting that the gyroscope may be more effective than the handlebar to control the vehicle roll in static conditions. However, this is not actually true because the gyroscope swing angle is constrained to remain small. As a consequence, the feedback controller must attenuate low frequencies and cannot be used to reach a static roll angle set-point rather than the equilibrium one. As far as speed is concerned, the variations of the steer torque to roll angle response function that occur for the reference motorcycle are replicated for both the single and twin gyroscopes. Moreover, the swing torque to roll angle response function is insensitive to the speed. This is not surprising, as the swing torque induces a gyroscopic effect which is applied directly on the chassis, while by contrast the steer torque to roll angle control is enforced trough tyre lateral forces, which depend on speed.

So far, the twin gyroscope configuration appears to be more suitable to preserve the vehicle original handling characteristic than the single gyroscope configuration. To further investigate this matter, figure 12 reports the steer torque to roll angle ratio (i.e. the reciprocal of the static of figure 11a) as a function of the speed. As already reported in [42], it may be observed that it is quite difficult to control the motorcycle at very low speed, and that the steering torque effort has a minimum at a relatively low speed 


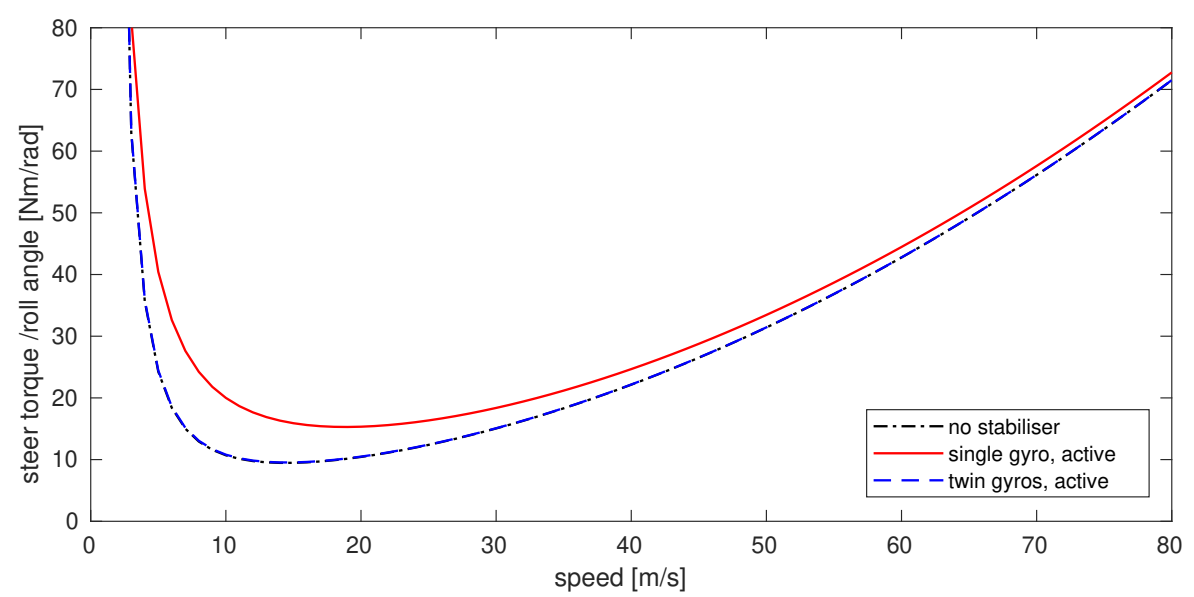

Figure 12. Steering torque to roll angle ratio in steady cornering.

and increases again with the speed. Even if the single gyroscopic stabiliser is not able to match perfectly this behavior as the twin gyroscopic stabiliser does, the increase in steering torque required in steady cornering is modest if the whole range of speed is considered. Indeed, the angular momentum of the gyroscope is constant and, as the speed increases, the angular momentum of the front wheel becomes dominant instead $[45,46]$.

Finally, the non-linear behavior of the motorcycle in transient and steady-state conditions is simulated in the time domain. Figure 13 gives the response of the gyroscope during cornering manoeuvres at speed of $15 \mathrm{~m} / \mathrm{s}$, when the active gyroscope stabiliser cooperates with the rider. With a twin gyroscope stabiliser, the cornering response in terms of steer angle, steer torque and roll angle is practically identical to that of the original vehicle. With a single gyroscope, a higher steering torque is required and the roll angle response is slightly delayed. In the twin gyroscope case, the gyroscopic swing torque has a peak value of approximately $80 \mathrm{Nm}$. Noting that in this case the peak value of gyroscope swing rate is around $2.0^{\circ} / \mathrm{s}$, we conclude that the gyroscopic actuation draws a peak power of approximately $9 \mathrm{~W}$. This power requirement is modest and could readily be supplied by either the internal combustion engine of a conventional vehicle or the battery and electric motor of an electric one. Although we have neglected the inefficiency of the electric motor and the power necessary to maintain the gyroscope spin motion, the power consumption is likely to be small even once these are taken into account.

\section{Conclusions}

The paper has explored the potential of gyroscopic systems for the stabilisation of the weave, wobble and capsize modes of motorcycles and single track vehicles more generally. First, different gyroscope configurations were examined, including different orientations of both the spinning and swinging (precessing) axes, and for both single and twin counterrotating gyroscopes. It has been shown that the most sensible configurations that should be used to stabilise a motorcycle are either a single gyroscope spinning with respect to an axis parallel to the wheels spin axis and swing with respect to the yaw axis, or a system of twin gyroscopes counter-rotating and counter-swinging with respect to the same axes. It has been also shown that the latter and a system composed by twin gyroscopes counterspinning with respect to the yaw axis and counter-swinging with respect to the pitch 

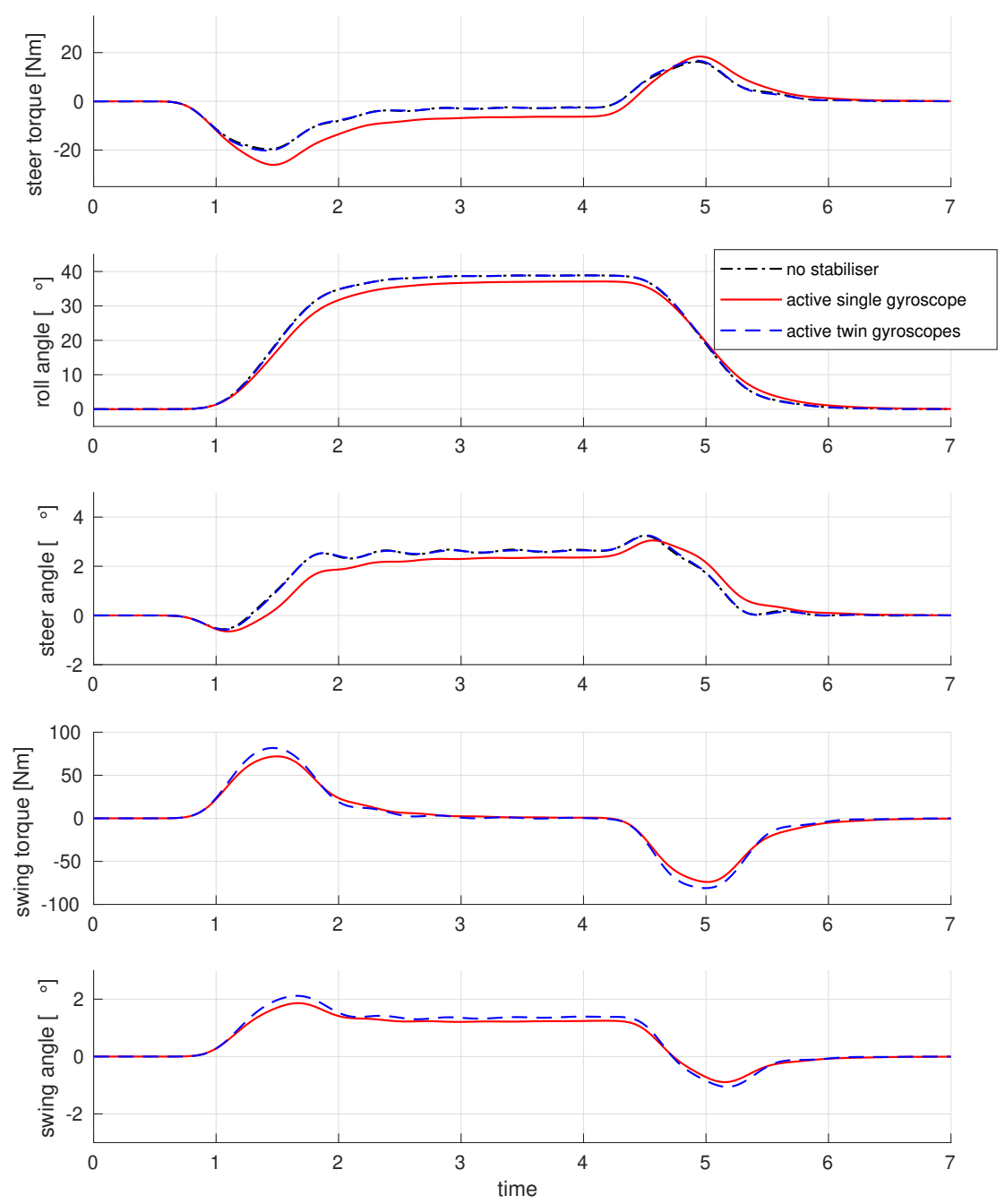

Figure 13. Motorcycle response under cornering, with active gyroscopic stabiliser, (motorcycle speed $15 \mathrm{~m} / \mathrm{s}$, steady cornering acceleration $a_{y}=6.3 \mathrm{~m} / \mathrm{s}^{2}$, transient roll rate peak $\omega_{\phi, \max }=46^{\circ} / \mathrm{s}$ ).

axis are completely equivalent. Any other configuration presented serious disadvantages.

Passive stabilisation systems, where the precession of the gyroscope is restrained by a spring and (possibly) a damper but is not actively controlled, were analysed first. It has been shown that such passive systems can effectively stabilise the vehicle at high speed, but are ineffectual at low speed. Cornering performance is only slightly affected by the presence of the stabiliser. However the necessity of adopting a "soft" swing spring to ensure stability may conflict with the necessity to limit the swing motion amplitude. The utilisation of a non-linear spring may effectively mitigate this problem. Indeed, the significance of non-linear effects in general on the stabilisation of the system has been found to be quite limited and conclusions drawn about stability and frequency response from linear analysis remain valid. In summary, considering the complexity of the system, which still fails to stabilise the vehicle at a low speed, we do not see a practical application 
for a passive gyroscopic stabiliser.

Active stabilisation systems, that is when the gyroscope motion is feedback controlled, have shown greater performance and in particular are capable of stabilising the motorcycle at almost zero speed and during braking. This characteristic could be very useful to improve driving comfort, for example by allowing the rider to avoid putting their feet down when riding very slowly or stopping at intersections, as well as being beneficial for safety, e.g. in combination with an emergency braking system to avoid - or at least mitigate - collisions. While we did not find a significant difference between a single active gyroscope and a twin counter-rotating gyroscope system in term of vehicle stabilisation, the latter performs better than the former in term of handling. Indeed a twin gyroscopic system may have practically no effect on the handling characteristics of the original vehicle. However, the handling differences for the single gyroscope case are appreciable only in the medium speed range and, considering the greater complexity of the twin gyroscopes versus the single one, it is not clear which of the two cases presents the more attractive option. In terms of control system, gain-scheduling LQR controllers seem to provide an effective control methodology. However, this assumes that the full state is available for feedback and so future work should consider output feedback strategies (for instance, using a state observer). In addition the robustness of the stabilisation to speed and acceleration changes requires further investigation, for example by construction of Lyapunov functions that account for the time-varying aspect of the system, as this is not guaranteed by the linear analysis carried out here.

\section{References}

[1] Sharp RS. The stability and control of motorcycles. Journal of mechanical engineering science. 1971; 13(5):316-329.

[2] Limebeer D, Evangelou S, Sharp R. Stability of motorcycles under acceleration and braking. Vol. 6 C; 2001. p. 2583-2591.

[3] Cossalter V, Lot R, Maggio F. The modal analysis of a motorcycle in straight running and on a curve. Meccanica. 2004;39(1):1-16.

[4] Cossalter V, Lot R, Massaro M. An advanced multibody code for handling and stability analysis of motorcycles. Meccanica. 2011;46(5):943-958.

[5] Marumo Y, Nagai M. Steering control of motorcycles using steer-by-wire system. User Modeling and User-Adapted Interaction. 2007;45(5):445-458.

[6] Benz C. Self-propelling vehicle. 385087. 1888.

[7] Summer TO. Gyro stabilized vehicle. Patent US 3410357. 1968.

[8] Hair JM. Gyro stabilized remote controlled toy motorcycle. Patent US 5820439. 1998.

[9] Kim DKY, Bretney K. Electrical system for gyroscopic stabilized vehicle. Patent US 2011/0231041 A1. 2011.

[10] Kim DKY. Dynamically balanced flywheel. Patent US 2013/0233100 Al. 2013.

[11] Klews M. Method and device for the driving stabilization of a motorized two-wheeled vehicle using a double-gyroscope device. Patent US 2015/0353151 Al. 2015.

[12] Beznos A, Formal'sky A, Gurfinkel E, Jicharev D, Lensky A, Savitsky K, Tchesalin L. Control of autonomous motion of two-wheel bicycle with gyroscopic stabilization. Vol. 3; 1998. p. 2670-2675.

[13] Karnopp D. Tilt control for gyro-stabilized two-wheeled vehicles. Vehicle System Dynamics. 2002; $37(2): 145-156$.

[14] Spry SC, Girard AR. Gyroscopic stabilisation of unstable vehicles: configurations, dynamics, and control. Vehicle System Dynamics. 2008;46(S1):247-260.

[15] Aoki A. Experimental study on motorcycle steering performance. Warrendale (PA): SAE; 1979 (TechnicalReport 790265). 1978;

[16] Katayama T, Aoki A, Nishimi T. Control behaviour of motorcycle riders. Vehicle System Dynamics. 1988;17(4):211-229.

[17] Kooijman J, Schwab A. A review on bicycle and motorcycle rider control with a perspective on handling qualities. Vehicle System Dynamics. 2013;51(11):1722-1764. 
[18] Aoki A, Nishimi T, Okayama T, Katayama T. Effectiveness of the basic model for motorcycle dynamics. Nihon Kikai Gakkai Ronbunshu, C Hen/Transactions of the Japan Society of Mechanical Engineers, Part C. 1999;65(636):3142-3148; cited By 2.

[19] Sharp R. Optimal linear time-invariant preview steering control for motorcycles. Vehicle System Dynamics. 2006;44(SUPPL. 1):329-340.

[20] Saccon A, Hauser J, Beghi A. A virtual rider for motorcycles: An approach based on optimal control and maneuver regulation; 2008. p. 243-248.

[21] Rowell S, Popov A, Meijaard J. Application of predictive control strategies to the motorcycle riding task. Vehicle System Dynamics. 2008;46(SUPPL.1):805-814; cited By 7.

[22] Lot R, Massaro M, Sartori R. Advanced motorcycle virtual rider. Vehicle System Dynamics. 2008; 46(SUPPL.1):215-224; cited By 13.

[23] Massaro M, Lot R. A virtual rider for two-wheeled vehicles; 2010. p. 5586-5591.

[24] Sharp R. Rider control of a motorcycle near to its cornering limits. Vehicle System Dynamics. 2012; 50(8):1193-1208; cited By 7.

[25] Zhu S, Murakami S, Nishimura H. Motion analysis of a motorcycle taking into account the rider's effects. Vehicle System Dynamics. 2012;50(8):1225-1245.

[26] Sequenzia G, Oliveri S, Fatuzzo G, Cal M. An advanced multibody model for evaluating rider's influence on motorcycle dynamics. Proceedings of the Institution of Mechanical Engineers, Part K: Journal of Multi-body Dynamics. 2015;229(2):193-207.

[27] Kamata Y, Nishimura H. System identification and attitude control of motorcycle by computer-aided dynamics analysis. JSAE Review. 2003;24(4):411-416.

[28] Cossalter V, Doria A, Formentini M, Peretto M. Experimental and numerical analysis of the influence of tyres properties on the straight running stability of a sport-touring motorcycle. Vehicle System Dynamics. 2012;50(3):357-375.

[29] Cossalter V, Lot R, Massaro M. The influence of frame compliance and rider mobility on the scooter stability. Vehicle System Dynamics. 2007;45(4):313-326.

[30] Kane T. The effect of frame flexibility on high speed weave of motorcycles. SAE Technical Papers. 1978 ;

[31] Verma M, Scott R, Segels L. Effect of frame compliance on the lateral dynamics of motorcycles. Vehicle System Dynamics. 1980;9(4):181-206; cited By 12.

[32] Koenen C, Pacejka H. Influence of frame elasticity, simple rider body dynamics and tyre moments on free vibrations of motorcycles in curves.; 1982. p. 53-65; cited By 15.

[33] Kageyama I, Kogo A. Human factors in the steering system of two-wheeled vehicles. Bulletin of the JSME. 1985;28(240):1233-1239.

[34] Cossalter V, Doria A, Lot R, Massaro M. The effect of rider's passive steering impedance on motorcycle stability: Identification and analysis. Meccanica. 2011;46(2):279-292.

[35] Massaro M, Lot R, Cossalter V, Brendelson J, Sadauckas J. Numerical and experimental investigation of passive rider effects on motorcycle weave. Vehicle System Dynamics. 2012;50(SUPPL. 1):215-227.

[36] Massaro M, Cole D. Neuromuscular-steering dynamics: Motorcycle riders vs car drivers. Vol. $3 ; 2012$. p. 217-224; cited By 8.

[37] Hoult W. A neuromuscular model for simulating driver steering torque; 2008.

[38] Lot R. A motorcycle tire model for dynamic simulations: Theoretical and experimental aspects. Meccanica. 2004;39(3):207-220.

[39] Pacejka H. Tire and vehicle dynamics. Elsevier; 2006.

[40] Lot R, Da Lio M. A symbolic approach for automatic generation of the equations of motion of multibody systems. Multibody System Dynamics. 2004;12(2):147-172.

[41] Lot R, Massaro M. A symbolic approach to the multi-body modelling of road vehicles. International Journal of Applied Mechanics. 2017, in printing;.

[42] Biral F, Bortoluzzi D, Cossalter V, Da Lio M. Experimental study of motorcycle transfer functions for evaluating handling. Vehicle System Dynamics. 2003;39(1):1-25.

[43] Rosenbrook H. The stability of linear time-dependent control systems. Journal of Electronics and Control. 1963;15(1):73-80.

[44] Desoer C. Slowly varying system $\mathrm{x}=\mathrm{a}(\mathrm{t}) \mathrm{x}$. IEEE Transactions on Automatic Control. 1969; 14(6):780-781.

[45] Cossalter V, Doria A, Lot R. Steady turning of two-wheeled vehicles. Vehicle System Dynamics. 1999;31(3):157-181.

[46] Cossalter V, Lot R, Peretto M. Steady turning of motorcycles. Proceedings of the Institution of Mechanical Engineers, Part D: Journal of Automobile Engineering. 2007;221(11):1343-1356. 